\title{
Finiteness of Mordell-Weil Groups of Kuga Fiber Spaces of Abelian Varieties
}

By

\author{
Masa-Hiko SAITO*
}

\begin{abstract}
In this paper, we will study Mordell-Weil groups of Kuga fiber spaces of abelian varieties associated to the standard symplectic representation classified by Satake. We will show the finiteness theorem for them with a few exceptions by using the Hodge theory and Borel-Wallach's vanishing theorem.
\end{abstract}

\section{$\S 0$. Introduction}

Let $f: \mathscr{X} \rightarrow M$ be a projective abelian scheme over an arithmetic quotient of a hermitian symmetric domain $M=\Gamma \backslash \mathscr{D}$, constructed from a symplectic representation of the associated algebraic group. Such fiber spaces of abelian varieties have been studied by Kuga, Shimura, Satake, Mumford, et al. Following Satake ([S1], Ch. IV), we call such a fiber space a Kuga fiber space (of abelian varieties). Let $\eta$ be the generic point of $M$ and $\mathscr{X}_{\eta}$ denotes the generic fiber of $f$. Then $\mathscr{X}_{\eta}$ can be considered as an abelian variety defined over the rational function field $K=\boldsymbol{C}(M)$, so define the Mordell-Weil group to be the group $\mathscr{X}_{\eta}(K)$ of $K$-rational points, or equivalently, the group of rational sections of $f: \mathscr{X} \rightarrow M$, and denote it by $M W(\mathscr{X} / M)$. In this paper, we shall study Mordell-Weil groups $M W(\mathscr{X} / W)$ of Kuga fiber spaces, and prove a finiteness theorem for them.

Historically, Shioda first showed that the Mordell-Weil groups of the elliptic modular surfaces corresponding to arithmetic subgroups $\Gamma \subset S L_{2}(\boldsymbol{Z})$ are finite in [Sd]. Generalizing Shioda's result, Silverberg [Si1] proved the finiteness of the Mordell-Weil groups of those Kuga fiber spaces which are characterized by an endomorphism algebra with positive involution and a polarization, introduced by Shimura in [Sh1] and [Sh2]. She later obtained in [Si2] a cohomological criterion for the finiteness, which covered the most of her former results.

Denote by $\boldsymbol{R}_{1} f^{*} \boldsymbol{C}_{\mathfrak{x}}$ the local system of the first homology groups of the

Communicated by Y. Ihara, July 15, 1991.

1991 Mathematics Subject Classification. primary: 14J10 secondary: 14G35, $14 \mathrm{G} 40$.

Supported in part by the Japan Foundation and JAMI of the Johns Hopkins University.

* Department of Mathematics, Faculty of Science, Kyoto University, Kyoto 606-01, Japan. 
fibers of $f$. Then the local system $\boldsymbol{R}_{1} f_{*} \boldsymbol{C}_{\mathfrak{X}}$ is induced by a representation $\Gamma \rightarrow G L\left(W_{\boldsymbol{c}}\right)$, and we have natural isomorphisms $H^{q}\left(M, \boldsymbol{R}_{1} f_{*} C_{\mathfrak{X}}\right) \cong H^{q}\left(\Gamma, W_{\boldsymbol{c}}\right)$ where $H^{q}\left(\Gamma, W_{c}\right)$ denotes the Eilenberg-MacLane cohomology group. The criterion of Silverberg says that if $\operatorname{dim} M>1$ or $M$ is compact and $H^{q}\left(\Gamma, W_{c}\right)=0$ for $q=0,1$ then the Mordell-Weil group $M W(\mathscr{X} / M)$ is finite.

This criterion directly works for the cases when the algebraic group $G_{\boldsymbol{Q}}$ defined over $\boldsymbol{Q}$ under consideration has rational rank $\geqq 2$, or the rational rank $=0$ (i.e. $\Gamma \subset G_{R}$ is cocompact) and $G_{R}$ has no compact factor and no factor isomorphic to $\operatorname{SU}(n, 1)$ (see Th. 6 and Th. 7 in [Si2], or [B-W]). (When the rational rank $=1$, see Theorem 7 of $[\mathrm{Si} 2]$ ).

On the other hand, there are examples of Kuga fiber spaces for which one can not apply these vanishing theorem directly, and in some cocompact cases, we do have examples with $H^{1}\left(\Gamma, W_{c}\right) \neq 0$. (See $\S 5$ ). But we can still expect the finiteness of the Mordell-Weil group (see [Si1], [Si3]).

As far as the classification of Kuga fiber spaces is concerned, Satake studied deeply $\boldsymbol{Q}$-symplectic representations, and classified all $\boldsymbol{Q}$-primary symplectic representations with a very mild additional condition ([S2], see also $\mathrm{IV}, \S 6$, [S1]), and every $\boldsymbol{Q}$-symplectic representation is a sum of primary represetations. They consist of the standard one which is constructed from the pair of a $D$ module $V$ with a $D$-skew hermitian or a $D$-hermitian form $h$ where $D$ is a division algebra over $\boldsymbol{Q}$ with center $F_{1}$, and the nonstandard one obtained from exterior product and spin representations. In the standard case, the $\boldsymbol{Q}$-algebraic group is given by $R_{F_{1} / Q}(S U(V, h))$, which is obtained from the $F_{1}$-algebraic group $S U(V, h)$ by Weil's restriction of the scalars. We remark that the standard representations include the cases which were studied by Shimura in [Sh3].

In this paper, we will only consider the standard $\boldsymbol{Q}$-symplectic representation. Also, we will exclude the following case from our consideration (cf.(3.42)):

Case $(\boldsymbol{R 2},-\mathbf{1}), \boldsymbol{n}=\mathbf{2}: G_{R} \cong S U_{2}(\boldsymbol{H})^{-} \times \cdots \times S U_{2}(\boldsymbol{H})^{-} \times S O_{4}(\boldsymbol{R}) \times \cdots \times S O_{4}(\boldsymbol{R})$,

because the reducibility of $S U_{2}(\boldsymbol{H})^{-}$forces annoying distinctions about the nature of $\Gamma$. (For the notation, see $\S 3,(3.23)$ and (3.31)).

Then the main theorem in this paper can be stated as follows.

(0.2) Theorem. ((4.23), (5.8) and (6.25)). Let $f: \mathfrak{X} \rightarrow M$ be a Kuga fiber space associated to a standard $\mathbf{Q}$-primary representation not isomorphic to the rase (0.1). Assume that $\operatorname{dim} M \geqq 1$. Then the Mordell-Weil group $M W(\mathfrak{X} / M)$ is finite.

The main idea of our proof is a generalization of Silverberg's method in [Si2] by introducing the $L_{2}$-cohomology and the Hodge theory, which can be 
outlined as follows.

If the codimension of the singular locus of the Satake compactification $M^{*}$ of $M$ is greater than 1 , then for $q \leqq 1, H^{q}\left(\Gamma, W_{\boldsymbol{C}}\right) \cong H^{q}\left(M, \boldsymbol{W}_{\boldsymbol{C}}\right)$ is isomorphic to the middle perversity intersection cohomology $\boldsymbol{I H}^{q}\left(M^{*}, \boldsymbol{W}_{C}\right)$. Then by the Zucker conjecture proved in $[\mathrm{L}]$ and $[\mathrm{Sa}-\mathrm{St}]$, these are also isomorphic to $L_{2}$-cohomology groups. By Borel-Casselman $[\mathrm{B}-\mathrm{C}]$, the $L_{2}$-cohomology is calculated by $(\mathrm{g}, K)$-cohomology, and hence we can apply the Borel-Wallach vanishing theorem in [B-W] even in the case when $\Gamma$ is not cocompact, and deduce that $H_{(2)}^{q}(M$, $\left.W_{C}\right)=0$ if $q<\operatorname{rank}_{\boldsymbol{R}} G_{\boldsymbol{R}}$. So if $\operatorname{rank}_{\boldsymbol{R}} G_{\boldsymbol{R}} \geqq 2$, we always have $H^{q}\left(M, \boldsymbol{W}_{\boldsymbol{C}}\right)=0$ for $q=0,1$. In case when $\operatorname{rank}_{R} G_{R}=1$, we will separate the proof into two cases, that is, the cases where $M=\Gamma \backslash \mathscr{D}$ is compact or non-compact.

If $M$ is compact, we can use Deligne-Zucker Hodge theory on $H^{q}\left(M, W_{C}\right)$, because $W_{C}$ admits a variation of polarized Hodge structure. It is proved that the Mordell-Weil group $M W(\mathscr{X} / M)$ is isomorphic to $H^{1}\left(M, \boldsymbol{W}_{\boldsymbol{Z}}\right) \cap\left(H^{0,0}\right)$ in this case. Since $W_{Q}$ has a structure of a local system of $F_{1}$-vector spaces, we have a decomposition of $\boldsymbol{W}_{\boldsymbol{C}}$ according to the distinct embeddings of $F_{1}$ into $\boldsymbol{C}$. We can see from Satake's classification that this decomposition is compatible with the Hodge structure. Though in this case it is possible that $H^{1}\left(M, \boldsymbol{W}_{C}\right)^{0,0} \neq 0$, we can use the decomposition of $H^{1}\left(M, \boldsymbol{W}_{C}\right)$ to conclude that $H^{1}\left(M, \boldsymbol{W}_{\boldsymbol{Q}}\right)^{0,0}=0$.

If $M$ is not compact and $\operatorname{rank}_{R} G_{R}=1$, we can take a smooth toroidal compactification $j: M \hookrightarrow \bar{M}$ such that $D=\bar{M}-M$ is a smooth divisor and consider the cohomology group $H^{1}\left(\bar{M}, j_{*} W_{\boldsymbol{Z}}\right)$. Then by a result due to Cattani-KaplanSchmid [C-K-S] and Kashiwara-Kawai [K-K], this admits polarized Hodge structure of weight 0 . On the other hand, we can extend the Kuga fiber space $f: \mathscr{X} \rightarrow M$ to a semi-abelian scheme $\bar{f}: \bar{X} \rightarrow \bar{M}$. And in this case one can prove that $\left.H^{0}\left(\bar{M}, \Theta_{\bar{M}} \frac{a n}{(\mathscr{X}}\right)\right)=H^{1}\left(\bar{M}, j_{*} \boldsymbol{W}_{\boldsymbol{Z}}\right)^{0,0}$, where $\left.H^{0}\left(\bar{M}, \Theta_{\bar{M}} \frac{a n}{(\mathscr{X}}\right)\right)$ denote the group of holomorphic sections of $\bar{f}$. By using the theory of Néron model, it can be shown that there is an injective homomorphism $r: H^{0}\left(M, \mathcal{O}_{\bar{y}}{ }^{n}(\overline{\mathcal{X}})\right) \hookrightarrow M W(\mathscr{X} / M)$ with finite cokernel. Now by using the description of Hodge structure due to Yuji Shimizu [ShzY], we calculate the Hodge component and we can finally prove that $H^{1}\left(\bar{M}, j_{*} W_{Q}\right)^{0,0}=0$.

The organization of this paper as follows. In $\S 1$, we introduce $\boldsymbol{Q}$-symplectic representations and Kuga fiber spaces. In $\S 2$, we introduce the MordellWeil groups of Kuga fiber spaces and recall some results due to Silverberg [Si1], [Si2]. We also review a Hodge theory of the cohomology group to give a slight refinement of Silverberg's results. In $\S 3$, we summarize the basic fact on Satake's classification of $\boldsymbol{Q}$-symplectic representations. In $\S 4$, we recall some results from Borel-Casselman [B-C] and Borel-Wallach [B-W], and prove the desired vanishing theorem when the $\boldsymbol{R}$-rank of $G_{\boldsymbol{R}} \geqq 2$, even if $G_{\boldsymbol{R}}$ has compact factors. In $\S 5$, we shall deal with the case when the $R$-rank of $G_{\boldsymbol{R}}$ is 1 and $M=\Gamma \backslash \mathscr{D}$ is compact. We will check that the decomposition (see (5.10)) is compatible with the Hodge structure, and we calculate the first Gauss-Manin 
complex whose $\boldsymbol{H}^{1}$ is the space of $(0,0)$-elements. In $\S 6$, we shall deal with the case when the $\boldsymbol{R}$-rank of $G_{\boldsymbol{R}}$ is 1 and $M$ is non-compact.

The author would like to thank Professor Steven Zucker for very useful discussions about Hodge theory, $L_{2}$-cohomology and intersection cohomology. He would also like to thank Professor Alice Silverberg for reading the preliminary version of this paper and giving useful comments. He would like to express his gratitude to JAMI in Johs Hopkins University for its hospitality during academic year 1990/91.

After I have finished the preliminary version of this paper, the author was informed that Ngaiming Mok announced the more general finiteness result of Moredell-Weil group of Kuga fiber spaces independently. It was announced in his preprint [Mo], though there were some gaps in their first version of full paper $[\mathrm{Mo}-\mathrm{T}]$. (They have assumed that $G_{\boldsymbol{R}}$ has no compact factor for all Kuga fiber spaces, which is not true in general.) They have fixed the gaps in the revised version of [Mo-T], which the author received after submission of this paper. The author believes that the method in this paper is different from theirs and it is worth while publishing this paper.

Notation. Let $T$ be a complex vector space. For a complex endomorphism $I$ and $\alpha \in C$, we set $T(\alpha, I)=\{u \in T \mid I(u)=\alpha \cdot u\}$, the eigenspace of $I$. We denote by $\boldsymbol{H}=\boldsymbol{R}+\boldsymbol{R} \cdot i+\boldsymbol{R} \cdot j+\boldsymbol{R} \cdot k$ the field of Hamilton quaternions.

\section{§1. Q-Symplectic Representations and Kuga Fiber Spaces}

Let $G_{\boldsymbol{Q}}$ be a $\boldsymbol{Q}$-algebraic group such that its $\boldsymbol{R}$-valued point $G_{\boldsymbol{R}}$ is a Zariski connected semisimple $\boldsymbol{R}$-group of hermitian type. Let $K$ be a maximal compact subgroup of $G_{R}$ and $\mathscr{D}=G_{R} / K$ the corresponding Hermitian bounded symmetric space. We denote by $\mathfrak{g}, \mathfrak{f}$ Lie algebras of $G_{R}$ and $K$ respectively, and by $\mathfrak{p}$ the orthogonal complement of $\mathfrak{f}$ in $g$ with respect to the Killing form. Then the complex structure of $\mathscr{D}$ is induced by an element $H_{0} \in \operatorname{Cent}(\mathfrak{f})$ such that $\left(\operatorname{ad}_{\mathfrak{p}}\left(H_{0}\right)\right)^{2}$

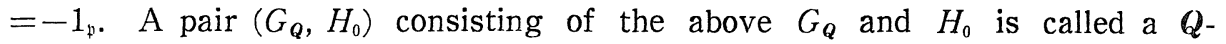
hermitian pair.

(1.1) Definition. A $\boldsymbol{Q}$-symplectic representation of a $\boldsymbol{Q}$-hermitian pair $\left(G_{\boldsymbol{Q}}\right.$, $\left.H_{0}\right)$ is a quadruples $\left(W_{Q}, \rho_{Q}, A_{Q}, I\right)$ consisting of

(i) a $\boldsymbol{Q}$-vector space $W_{\boldsymbol{Q}}$ of dimension $n$,

(ii) a non-degenerate symplectic bilinear form $A_{Q}$ on $W_{Q} \times W_{Q}$,

(iii) a faithful representation $\rho_{Q}: G_{Q} \rightarrow S p\left(W_{Q}, A_{Q}\right)$ and

(iv) a complex structure $I \in \mathscr{D}\left(W_{R}, A_{R}\right)$ satisfying the condition

$$
\left[d \rho_{R}\left(H_{0}\right)-(1 / 2) I, d \rho_{R}(X)\right]=0 \quad \text { for all } X \in g_{R},
$$


where $\mathscr{D}\left(W_{R}, A_{R}\right)$ denotes

(1.3) $\left\{I \in \operatorname{End}\left(W_{R}\right) \mid I^{2}=-1_{W_{R}}, A_{R}(x, I y)\right.$ is a positive-definite $\boldsymbol{R}$-symmetric form $\}$.

(See (3.11)).

Next we introduce a Kuga fiber space of abelian varieties induced from a $\boldsymbol{Q}$-symplectic representation. Let $\left(W_{\boldsymbol{Q}}, \rho_{\boldsymbol{Q}}, A_{\boldsymbol{Q}}, I\right)$ be a $\boldsymbol{Q}$-symplectic representation of a $\boldsymbol{Q}$-hermitian pair $\left(G_{\boldsymbol{Q}}, H_{0}\right)$. By a lattice in $W_{\boldsymbol{Q}}$, we mean a free $\boldsymbol{Z}$ submodule $W_{\boldsymbol{Z}}$ in $W_{\boldsymbol{Q}}$ such that $W_{\boldsymbol{Z}} \otimes_{\boldsymbol{Z}} \boldsymbol{Q} \cong W_{\boldsymbol{Q}}$. Considering $G_{\boldsymbol{Q}}$ as a subgroup in $G L\left(W_{Q}\right)$ through the representation $\rho_{Q}: G_{Q} \rightarrow S p\left(W_{Q}, A_{Q}\right)$, for each lattice $W_{Z}$ in $W_{Q}$, we set

$$
G_{W_{Z}}=\left\{g \in G_{\boldsymbol{Q}} \mid g W_{Z}=W_{Z}\right\} .
$$

Then $G_{W_{Z}} \subset G_{Q}$ becomes a discrete subgroup of $G_{R}$.

(1.5) Definition-Proposition. ([S1, Ch. IV, §7]). A discrete subgroup $\Gamma$ of $G_{R}$ commensurable to $G_{W_{Z}}$ for some lattice $W_{Z}$ is called an arithmetic subgroup of $G_{R}$. The quotient space $\Gamma \backslash G_{R}$ is of finite measure with respect the measure induced from the Haar measure of $G_{R}$, and there always exists a normal subgroup $\Gamma^{\prime}$ of $\Gamma$ of finite index such that $\Gamma^{\prime}$ is torsion-free.

(1.6) Definition. A 5-tuple $\left(W_{Q}, \rho_{Q}, A_{Q}, I, W_{Z}\right)$ is said to be a Kuga 5-tuple if $\left(W_{Q}, \rho_{Q}, A_{Q}, I\right)$ is a $\boldsymbol{Q}$-symplectic representation of a $\boldsymbol{Q}$-hermitian pair $\left(G_{\boldsymbol{Q}}\right.$, $H_{0}$ ) and $W_{Z}$ is lattice of $W_{Q}$ such that

$$
A_{Q}\left(W_{Z}, W_{Z}\right) \subset Z \text {. }
$$

From a Kuga 5-tuple, we obtain a fiber space of abelian varieties as follows. Let $K$ be the maximal compact subgroup of $G_{R}$ determined by $H_{0}$, and denote by $\mathscr{D}=G_{\boldsymbol{R}} / K$ the corresponding hermitian symmetric space. Set $W_{\boldsymbol{R}}=W_{\boldsymbol{Q}} \otimes_{\boldsymbol{Q}} \boldsymbol{R}$, $W_{C}=W_{Q} \otimes_{Q} C$. We have a complex structure $I_{0} \in \mathscr{D}\left(W_{R}, A_{R}\right)$ (cf. (1.3)) satisfying (1.2). For an element $g \in G_{R}$, define

$$
I_{g}=\rho^{-1}(g) \cdot I \cdot \rho(g) .
$$

Then, by definition, we have $I_{g} \in \mathscr{D}\left(W_{R}, A_{R}\right)$, and from (1.2), $I_{g}=I_{0}$ for $g \in K$. Hence we define, for each point $z=[g] \in \mathscr{D}=G_{R} / K$,

$$
I_{z}=I_{g} \in \mathscr{D}\left(W_{R}, A_{R}\right) .
$$

Setting $W_{z}^{+}=\left\{u \in W_{C} \mid I_{z} u=\sqrt{-1} u\right\}$, we can obtain a holomorphic vector bundle $\tilde{\mathcal{F}}^{0}=\cup_{z \in \mathscr{D}} W_{z}^{+}$over $\mathscr{D}$ such that the following diagram commutes.

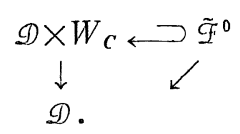


Let $\Gamma$ be a torsion-free arithmetic subgroup of $G_{R}$ such that $\Gamma \subset G_{W_{Z}}$. Then the quotient space $M=\Gamma \backslash \mathscr{D}$ is a complex manifold, which is known to be a quasi-projective variety $([\mathrm{Ba}-\mathrm{B}])$. Denote by $W_{\boldsymbol{Z}}$ the local system of free $Z$-modules on $M$ induced by the flat bundle $\left(\mathscr{D} \times W_{Z} / \sim\right)$, where $\sim$ denotes the equivalence relation given by

$$
(z, w) \sim(\gamma \cdot z, \rho(\gamma) \cdot v) \quad \text { for } \quad \gamma \in \Gamma .
$$

We also denote by $\boldsymbol{W}_{\boldsymbol{Q}}, \boldsymbol{W}_{\boldsymbol{R}}, \boldsymbol{W}_{\boldsymbol{C}}$ the local systems on $M$ corresponding to $W_{Q}$, $W_{R}, W_{C}$ respectively. The $G_{Q}$-invariant form $A_{Q}$ induces a flat symplectic bilinear form $A$ on $W_{Q}$. A holomorphic vector bundle $\tilde{F}^{0}$ on $\mathscr{D}$ descends to $M$ and we denote by $\mathscr{F}^{0}$ the corresponding locally free sheaf on $M$. Now we have the following

(1.10) Definition-Proposition. The triple $\left(\boldsymbol{W}_{\boldsymbol{Z}}, \boldsymbol{A}, \mathscr{F}^{0}\right)$ constructed above becomes a variation of polarized Hodge structure (VPHS, for short) of weight -1 , and of types $(-1,0),(0,-1)$ over $M=\Gamma \backslash \mathscr{D}$, i.e.,

(i) $\boldsymbol{A}$ is a flat $\boldsymbol{Z}$-valued non-degenerate symplectic form on $\boldsymbol{W}_{Z}$,

(ii) $\mathscr{F}^{0} \subset W_{Z} \otimes_{Z} \mathcal{O}_{M}$ defines a Hodge filtration of weight -1 , and of types $(-1,0),(0,-1)$, i.e.

$$
0=\mathscr{F}^{1} \subset \mathscr{F}^{0} \subset \mathscr{F}^{-1}=W_{Z} \otimes_{Z} \mathcal{O}_{M},
$$

such that

$$
\mathscr{F}^{0} \oplus \overline{\mathscr{F}}^{0} \cong W_{Z} \otimes_{Z} \mathcal{O}_{M}
$$

(iii) $\boldsymbol{A}$ satisfies the Hodge-Riemann bilinear relations, i.e. for a non-zero local section $u \in \mathscr{F}^{0}$, we have

$$
\begin{gathered}
A(u, u)=0, \\
-(\sqrt{-1}) A(u, \bar{u})>0 .
\end{gathered}
$$

As explained in $[\mathrm{D} 2,(4.4 .3)]$, we have an equivalence between the category of polarized abelian schemes over $M$ and the category of variations of polarized Hodge structure over $M$ of weight -1 , and of types $(-1,0),(0,-1)$, so we obtain a fiber space $f: \mathscr{X} \rightarrow M$ of abelian varieties over $M$.

(1.11) Definition-Proposition. ([S1, Ch. IV, § 8], or [Sh2, 3.10].) A fiber space of abelian varieties $f: \mathscr{X} \rightarrow M=\Gamma \backslash \mathscr{D}$ obtained from a Kuga 5-tuple $\left(W_{Q}\right.$, $\rho_{\boldsymbol{Q}}, A_{\boldsymbol{Q}}, I, W_{\boldsymbol{Z}}$ ) and a torsion-free arithmetic subgroup $\Gamma \subset G_{W_{\boldsymbol{Z}}}$ of $G_{\boldsymbol{R}}$ is called a Kuga fiber space (of abelian varieties). The total space $\mathfrak{X}$ is a smooth quasiprojective variety and $f$ is a smooth projective morphism.

\section{§. A Criterion of Silverberg and a Generalization}

In this section, we review a criterion of the finiteness of Mordell-Weil 
group of Kuga fiber spaces due to Silverberg [Si2], and give a slight generalization.

First of all, we introduce the Mordell-Weil group of a fiber space of abelian varieties. Let $M$ be a connected smooth quasi-projective variety. By a fiber space of abelian varieties over $M$ we mean a polarized smooth abelian scheme $f: \mathfrak{X} \rightarrow M$. Consider the generic fiber $\mathscr{X}_{\eta}$ of $f$. Then $\mathscr{X}_{\eta}$ is considered as an abelian variety over the field $K=\boldsymbol{C}(M)$ of the rational functions on $M$. Then the Mordell-Weil group of $f$ is defined to be the group of $K$-rational points $\mathscr{X}_{\eta}(K)$, and is denoted by $M W(\mathfrak{X} / M)$. There exists a natural isomorphism

$$
M W(\mathscr{X} / M)=\{\text { a rational section } s: M \cdots \rightarrow \mathscr{X} \text { of } f\} .
$$

Now let $\left(W_{\boldsymbol{Q}}, \rho_{\boldsymbol{Q}}, A_{\boldsymbol{Q}}, I, W_{\boldsymbol{Z}}\right)$ be a Kuga 5 -tuple for a $\boldsymbol{Q}$-hermitian pair $\left(G_{Q}, H_{0}\right), \Gamma \subset G_{W_{Z}}, M=\Gamma \backslash \mathscr{D}$ as in $\S 1$, and $f: \mathscr{X} \rightarrow M$ the associated Kuga fiber space (see (1.11)). Let $\mathcal{O}_{M}(\mathscr{X})$ (resp. $\mathcal{O}_{M}^{a n}(\mathscr{X})$ ) denote the sheaf of germs of regular algebraic (resp. holomorphic) sections with values in $\mathscr{X}$ (resp. $\mathscr{X}^{a n}$ ). The cohomology group $H^{0}\left(M, \mathcal{O}_{M}(\mathscr{X})\right)$ is isomorphic to the group of regular algebraic sections of $f$. A rational section $s \in M W(\mathscr{X} / M)$ always extends to a regular algebraic section in case of a Kuga fiber space (see [Sil, Prop. 2.1]). So we have

(2.2) Proposition. For a Kuga fiber space $f: \mathfrak{x} \rightarrow M$, we have an isomorphism

$$
M W(\mathfrak{X} / M) \cong H^{0}\left(M, \mathcal{O}_{M}(\mathscr{X})\right) .
$$

(2.4) Remark. From the construction, there exists a natural map

$$
H^{0}\left(M, \mathcal{O}_{M}(\mathfrak{X})\right) \longrightarrow H^{0}\left(M, \mathcal{O}_{M}^{a n}(\mathfrak{X})\right) .
$$

In general, there exists a holomorphic section of $f$ which is not algebraic, (e.g. consider the case where $M$ is a non-compact curve.) Assume that $\Gamma$ is irreducible in $G_{R}$ (see (4.4)). Then, if either $\operatorname{dim}(\mathscr{D})>1$, or $M$ is compact, one can show that (2.5) must be an isomorphism (see [Si2, §1], [Ba-B, §10]).

For $\boldsymbol{K}=\boldsymbol{Z}, \boldsymbol{Q}, \boldsymbol{R}, \boldsymbol{C}$, let $H^{\cdot}\left(\Gamma, W_{\boldsymbol{K}}\right)$ denote the Eilenberg-MacLane cohomology groups induced by the representation $\rho_{Q}$ and an arithmetic group $\Gamma$. Since $\mathscr{D}$ is contractible, we have natural isomorphisms for $K=\boldsymbol{Z}, \boldsymbol{Q}, \boldsymbol{R}, \boldsymbol{C}$

$$
H \cdot\left(\Gamma, W_{K}\right) \cong H \cdot\left(M, W_{\boldsymbol{K}}\right)
$$

where $\boldsymbol{W}_{\boldsymbol{K}}$ denote the local system on $M$ associated to $W_{K}$, (see (1.9)).

Now we can state the Silverberg's criterion of the finiteness of $M W(\mathscr{X} / M)$ ([Si2, Theorem 5]).

(2.7) Theorem. Assume that $\Gamma$ is irreducible (cf. (4.4)) and $\operatorname{dim} \mathscr{D}>1$ or $M=\mathscr{D} / \Gamma$ is compact. If 


$$
H^{0}\left(\Gamma, W_{c}\right)=H^{1}\left(\Gamma, W_{c}\right)=0,
$$

the Mordell-Weil group $M W(\mathfrak{X} / M)$ is finite, and isomorphic to $H^{1}\left(\Gamma, W_{Z}\right) \cong$ $H^{1}\left(M, W_{z}\right)$.

\section{(2.9) $L_{2}$-cohomology}

Let $f: \mathscr{X} \rightarrow M=\Gamma \backslash \mathscr{D}$ be a Kuga fiber space as above, and $\left(\boldsymbol{W}_{\boldsymbol{Z}}, \boldsymbol{A}, \mathscr{F}^{0}\right)$ the corresponding VPHS of types $(0,-1),(-1,0)$ as in (1.10).

The local system $W_{C}=W_{Z} \otimes_{Z} C$ has a flat symmetric bilinear form $A_{C}$, and if we denote by $C_{z}$ the Weil operator, (or the complex structure) of a fiber $\boldsymbol{W}_{\boldsymbol{C}}$, the form $T_{z}(x, y):=\boldsymbol{A}_{\boldsymbol{C}}\left(x, C_{z} \bar{y}\right)$ becomes a positive-definite hermitian form, so it induces a metric on $\boldsymbol{W}_{C}$. From the construction of $W_{C}$ and $\boldsymbol{A}_{C}$, this metric is nothing but the one induced by the admissible inner product on $W_{C}$ ([M-M, p 375]). The base space $M=\Gamma \backslash \mathscr{D}$ is endowed with a complete metric induced by the Bergman metric on $\mathscr{D}$. Hence, we can give a norm on each term of the complex $A \cdot\left(M, W_{C}\right)^{\infty}$ of $W_{C}$-valued $C^{\infty}$ exterior forms on $M$. Let $L_{(2)}\left(M, W_{C}\right)^{\infty}$ denote its subcomplex consisting of square-integrable elements whose exterior derivative are also square-integrable. We define the $L_{2}$-cohomology group for $W_{C}$ by

$$
H_{(2)}\left(M, W_{C}\right):=H \cdot\left(L_{(2)}\left(M, W_{C}\right)^{\infty}\right) \text {. }
$$

Let $M^{*}$ denote the Baily-Borel, Satake compactification of $M$. It is known that $M^{*}$ is a normal projective variety which has a stratification by complex subvarieties. Following [G-M], we can define the middle perversity intersection cohomology group $\boldsymbol{I H} \cdot\left(M^{*}, \boldsymbol{W}_{C}\right)$. The following theorem is a direct consequence of the result, which was known as the Zucker conjecture, proved by Looijenga [L] and Saper-Stern [Sa-St].

(2.11) Theorem. Under the notation and assumption as above, we have isomorphisms

$$
H_{(2)}\left(M, \boldsymbol{W}_{C}\right) \cong \boldsymbol{I} \boldsymbol{H} \cdot\left(M^{*}, \boldsymbol{W}_{C}\right) .
$$

(2.12) Corollary. If $\operatorname{codim}_{C}\left(M^{*}-M\right)=i$ in $M^{*}$, then we have isomorphisms

$$
H_{(2)}^{q}\left(M, W_{C}\right) \cong H^{q}\left(M, W_{C}\right) \quad \text { for } q<i .
$$

Proof. From the definition of the intersection cohomology group [G-M, $\S 3,3.1]$, one can easily deduce that

$$
\boldsymbol{I} \boldsymbol{H}^{q}\left(M, \boldsymbol{W}_{C}\right) \cong H^{q}\left(M, \boldsymbol{W}_{C}\right) \quad \text { for } q<i,
$$

hence (2.11) implies the assertion.

If $\Gamma$ is irreducible in $G_{R}$ (see (4.4)) and $\operatorname{dim} \mathscr{D}>1$, one has $\operatorname{codim}_{C}\left(M^{*}-M\right)$ 
$\geqq 2$ in $M^{*}$. Hence, thanks to $(2.12)$, we have the following

(2.13) Corollary. Assume that $\Gamma \subset G_{R}$ is irreducible and $\operatorname{dim} \mathscr{D}>1$, or $M=$ $\Gamma \backslash \mathscr{D}$ is compact. Then there exist isomorphisms

$$
H_{(2)}^{q}\left(M, W_{C}\right) \cong H^{q}\left(M, W_{C}\right) \quad \text { for } \quad q \leqq 1 .
$$

\section{(2.15) Hodge theory in case $M$ is compact}

We recall that the triple $\left(\boldsymbol{W}_{\boldsymbol{Z}}, \boldsymbol{A}, \mathscr{F}^{0}\right)$ constructed in $\S 1$ is a VPHS of weight -1 of types $(0,-1),(-1,0)$ (see $(1.10))$. In particular, the sheaf $\boldsymbol{W}_{\boldsymbol{O}}:=$ $\boldsymbol{W}_{\boldsymbol{Z}} \otimes_{\boldsymbol{Z}} \mathcal{O}_{M}$ has a Hodge filtration

$$
0=\mathscr{F}^{1} \subset \mathscr{F}^{0} \subset \mathscr{F}^{-1}=\boldsymbol{W}_{\mathrm{O}} .
$$

Assume now that $M=\Gamma \backslash \mathscr{D}$ is compact. Then we have an isomorphism

$$
H^{n}\left(M, W_{C}\right) \cong H_{(2)}^{n}\left(M, W_{C}\right) \text { for all } n \text {. }
$$

In this case, from the $L_{2}$-harmonic theory, the right hand side of (2.16) can be expressed as a space of $\boldsymbol{W}_{\boldsymbol{C}}$-valued $L_{2}$-harmonic forms. Deligne showed that, as in the classical Hodge theory, there exists a decomposition

$$
H^{n}\left(M, W_{C}\right) \cong H_{(2)}^{n}\left(M, W_{C}\right)=\bigoplus_{p+q=n-1} H^{p, q}
$$

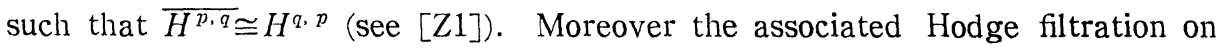
$H^{n}\left(M, W_{C}\right)$ is given as follows. Let $\Omega_{M}\left(\boldsymbol{W}_{C}\right)$ denote the holomorphic de Rham complex with values in $W_{C}$, with differential $\partial_{M}$. If we define the filtration $\left(F^{r} \Omega_{M}\left(\boldsymbol{W}_{C}\right)\right)$ by

$$
F^{r} \Omega_{M}^{p}\left(\boldsymbol{W}_{C}\right)=\Omega_{M}^{p} \otimes \mathscr{I}^{r-p},
$$

Griffiths' transversality (see e.g. [Z1]) implies that they actually become subcomplexes of $\Omega_{M}\left(\boldsymbol{W}_{C}\right)$. The holomorphic Poincaré lemma implies that

$$
H \cdot\left(M, \boldsymbol{W}_{C}\right) \cong \boldsymbol{H} \cdot\left(\Omega_{\dot{M}}\left(\boldsymbol{W}_{\boldsymbol{C}}\right)\right),
$$

and the above filtration induces a filtration on the cohomology.

(2.18) Theorem. Under the above notation, we have the following.

(i) The spectral sequence

$$
E_{1}^{p, q}=\boldsymbol{H}^{p+q}\left(M, G r_{F}^{p} Q_{M}^{\cdot}\left(\boldsymbol{W}_{C}\right)\right) \Longrightarrow H^{p+q}\left(M, \boldsymbol{W}_{C}\right)
$$

degenerates at $E_{1}$.

(ii) The filtration induced by $\left\{F^{p} \Omega_{M_{M}}\left(\boldsymbol{W}_{C}\right)\right\}$ on $H^{n}\left(M, W_{C}\right)$ coincides with the Hodge filtration induced from the decomposition (2.17).

(iii) There is a natural identification

$$
H^{p, q} \cong \boldsymbol{H}^{n}\left(M, G r_{F}^{p} \Omega_{M}\left(\boldsymbol{W}_{C}\right)\right)
$$


for $p+q=n-1$.

(iv) The cohomology group $H^{n}\left(M, \boldsymbol{W}_{\boldsymbol{Z}}\right) /$ torsion is a $\boldsymbol{Z}$-structure of $H^{n}\left(M, \boldsymbol{W}_{C}\right)$, and has a natural polarization $\boldsymbol{B}$, i.e. a $\boldsymbol{Z}$-valued bilinear form satisfying the Hodge-Riemann bilinear relations.

For example, $H^{0}\left(M, W_{C}\right)$ has a 2-step filtration $0=F^{1} \subset F^{0} \subset F^{-1}$ whose successive quotients are:

$$
\begin{aligned}
& H^{0,-1}=G r_{F}^{0}=F^{0}=H^{0}\left(\mathscr{F}^{0} \longrightarrow \Omega_{M} \otimes G r_{\mathscr{q}}^{-1}\right), \\
& H^{-1,0}=G r_{F}^{-1}=F^{-1} / F^{0}=\boldsymbol{H}^{0}\left(G r_{\bar{q}}^{-1}\right) .
\end{aligned}
$$

where $G r_{\mathscr{I}}^{-1}=\mathscr{F}^{-1} / \mathscr{F}^{0} . \quad H^{1}\left(M, W_{C}\right)$ has a 3-step filtration $0=F^{2} \subset F^{1} \subset F^{0} \subset F^{-1}=$ $H^{1}$ whose successive quotients are:

$$
\begin{aligned}
& H^{1,-1}=G r_{F}^{1}=F^{1}=H^{1}\left(0 \longrightarrow \Omega_{M}^{1} \otimes \mathscr{F}^{0} \longrightarrow \Omega_{M}^{2} \otimes G r_{\Phi}^{-1}\right), \\
& H^{0,0}=G r_{F}^{0}=F^{0} / F^{1}=H^{1}\left(\mathscr{F}^{0} \longrightarrow \Omega_{M}^{1} \otimes G r_{\Phi}^{-1}\right), \\
& H^{-1,1}=G r_{F}^{-1}=F^{-1} / F^{0}=\boldsymbol{H}^{1}\left(G r_{\Psi}^{-1}\right) .
\end{aligned}
$$

Considering $H^{1}\left(M, W_{\boldsymbol{Q}}\right)$ as a lattice of $H^{1}\left(M, \boldsymbol{W}_{C}\right)$, we set

$$
H^{1}\left(M, W_{Q}\right)^{0,0}=H^{1}\left(M, W_{Q}\right) \cap H^{0,0} .
$$

Let $p_{n}: H^{n}\left(M, W_{C}\right) \rightarrow H^{-1, n}=H^{n}\left(M, G r_{q}^{-1}\right)$ be the natural projection map induced by the spectral sequence (2.19). Set also

$$
\begin{gathered}
X_{\text {const }}=\operatorname{coker}\left\{p_{0}: H^{0}\left(M, \boldsymbol{W}_{\boldsymbol{Z}}\right) \rightarrow H^{0}\left(G r_{\bar{F}}^{-1}\right)\right\}, \\
H^{1}\left(M, \mathbb{W}_{\boldsymbol{Z}}\right)^{0,0}=\operatorname{ker}\left\{p_{1}: H^{1}\left(M, \boldsymbol{W}_{\boldsymbol{Z}}\right) \rightarrow H^{1}\left(M, G r_{\Phi}^{-1}\right)\right\} .
\end{gathered}
$$

Then by Hodge theory (2.18), one has

$$
H^{1}\left(M, W_{Q}\right)^{0,0}=H^{1}\left(M, W_{Z}\right)^{0,0} \otimes_{Z} \boldsymbol{Q} .
$$

Under these notations, we can state the following theorem which gives a very natural description of $M W(\mathfrak{X} / M)$. (Cf. [Z1, Cor. 10.2].)

(2.27) Theorem. Assume that $M=\Gamma \backslash \mathscr{D}$ is compact. Then

(i) $X_{\text {const }}$ in (2.24) is an abelian variety over $\boldsymbol{C}$, and

(ii) we have a natural exact sequence of abelian group

$$
0 \longrightarrow X_{\text {const }} \longrightarrow M W(\mathscr{X} / M) \longrightarrow H^{1}\left(M, W_{Z}\right)^{0,0} \longrightarrow 0 \text {. }
$$

Proof. The assertion ( $\mathrm{i}$ ) is an immediate consequence of (2.18). Since $M$ is projective, all holomorphic sections become algebraic, so by (2.5), we have an isomorphism $M W(\mathscr{X} / M) \cong H^{0}\left(M, \mathcal{O}_{M}^{a n}(\mathfrak{X})\right)$. The relative exponential map for an abelian scheme $f: \mathscr{X} \rightarrow M$ yields the following exact sequence of sheaf of $M^{a n}$

$$
0 \longrightarrow \boldsymbol{R}_{1} f_{*} \boldsymbol{Z} \longrightarrow \operatorname{Lie}(\mathfrak{X}) \longrightarrow \mathcal{O}_{M}^{a n}(\mathfrak{X}) \longrightarrow 0,
$$


where $\boldsymbol{R}_{1} f_{*} \boldsymbol{Z}$ denote the local system of the first homology of fibers of $f$. From the construction of a Kuga fiber space, we have isomorphisms $\boldsymbol{W}_{\boldsymbol{Z}} \cong \boldsymbol{R}_{1} f_{*} \boldsymbol{Z}$ and $\operatorname{Lie}(\mathscr{X}) \cong G r_{\bar{g}}^{-1}$, hence $\left(^{*}\right)$ can be written as

$$
0 \longrightarrow \boldsymbol{W}_{\boldsymbol{Z}} \longrightarrow G r_{\bar{F}}^{-1} \longrightarrow \mathcal{O}_{M}^{a n}(\mathfrak{X}) \longrightarrow 0 .
$$

This yields an exact sequence of cohomology group

$$
\begin{aligned}
0 & \longrightarrow H^{0}\left(M, W_{Z}\right) \stackrel{p_{0}}{\longrightarrow} H^{0}\left(M, G r_{\Phi}^{-1}\right) \longrightarrow H^{0}\left(M, \mathcal{O}_{M}^{a^{n}}(\mathfrak{X})\right) \\
& \longrightarrow H^{1}\left(M, \boldsymbol{W}_{\boldsymbol{Z}}\right) \stackrel{p_{1}}{\longrightarrow} H^{1}\left(M, G r_{\Phi}^{-1}\right),
\end{aligned}
$$

from which (2.28) follows.

q.e.d.

As a corollary, we have the following generalization of Silverberg's result (2.7).

(2.31) Theorem. Assume that $\Gamma \backslash \mathcal{D}$ is compact. The Mordell-Weal group $M W(\mathfrak{X} / M)$ of a Kuga fiber space is finite if and only if

$$
H^{0}\left(M, G r_{\tilde{q}}^{-1}\right)=H^{1}\left(M, W_{Q}\right)^{0,0}=0 .
$$

\section{$\S 3$. Satake's Classification of Q-Symplectic Representations}

In this section, we will summarize the Satake's work of classification of $Q$-symplectic representations. The main references are [S1], [S2].

\section{(3.1) Preliminary}

Let $F$ be a field of characteristic zero and $D$ a division algebra over $F$. Denoting by $F_{1}$ the center of $D$, we set

$$
\left[F_{1}: F\right]=d, \quad\left[D: F_{1}\right]=r^{2} .
$$

Consider a finite dimensional $F$-vector space $V$ with a structure of a right $D$ module, and set $n=\operatorname{rank}_{D} V$. We set:

$$
\begin{aligned}
& G L(V / D)=\left\{g \in \operatorname{End}_{D}(V) \mid g \text { is invertible }\right\}, \\
& S L(V / D)=\{g \in G L(V / D) \mid N(g)=1\},
\end{aligned}
$$

where $N$ denote the reduced norm of $\operatorname{End}_{D}(V)$. The corresponding matrix group are denoted by $G L_{n}(D)$ and $S L_{n}(D)$ respectively.

Let $\iota$ be an involution on $D$ and let $\varepsilon= \pm 1$. A $(D, \varepsilon)$-hermitian form $h$ on $V$ with respect to $\iota$ is by definition a $F$-bilinear mapping $h: V \times V \rightarrow D$ satisfying the following conditions :

$$
\begin{aligned}
& h\left(v, v^{\prime} \alpha\right)=h\left(v, v^{\prime}\right) \alpha, \\
& h\left(v^{\prime}, v\right)=\varepsilon h\left(v, v^{\prime}\right)^{c} \quad \text { for all } \quad v, v^{\prime} \in V, \alpha \in D .
\end{aligned}
$$


A $(D, \varepsilon)$-hermitian form $h$ is called non-degenerate if an intersection matrix $T=\left(h\left(e_{i}, e_{j}\right)\right)$ for a $D$-basis $\left(e_{i}\right)$ of $V$ is invertible. Fix an involution $\iota$ on $D$. For a non-degenerate $(D, \varepsilon)$-hermitian form $h$ on $V$ with respect to $\iota$, we define the unitary group and the special unitary group for $h$ by

$$
\begin{aligned}
& U(V, h)=\left\{g \in G L(V / D) \mid h\left(g v, g v^{\prime}\right)=h\left(v, v^{\prime}\right),\left(v, v^{\prime} \in V\right)\right\} \\
& S U(V, h)=U(V, h) \cap S L(V / D),
\end{aligned}
$$

and the corresponding matrix group are denoted by $U_{n}(D, h)$ and $S U_{n}(D, h)$ respectively.

The groups $G L_{n}(D), S L_{n}(D), U_{n}(D, h)$ and $S U_{n}(D, h)$ can be viewed as algebraic group defined over $F_{1}$. For a general $F_{1}$-group $G$, we denote by $R_{F_{1} / F}(G)$ the $F$-group obtained by scalar restriction (Weil $[\mathrm{W}, 1.3]$ ).

\section{(3.7) Classical groups over $\boldsymbol{R}$ and classical domains}

If $F=\boldsymbol{R}$, we can define the classical groups and classical domains of type (I), (II), (III). A division algebra $D$ over $\boldsymbol{R}$ must be either $\boldsymbol{R}, \boldsymbol{C}$, or $\boldsymbol{H}$, and here let $c$ be the standard involution of $D$.

Let $h$ be a non-degenerate skew-hermitian form on $V$ (i.e. $(D,-1)$-hermitian form) with respect to $c$. We can find a $D$-basis $\left(e_{i}\right)$ for $V$ such that the corresponding matrix $T=\left(h\left(e_{i}, e_{j}\right)\right) \in M_{n}(D)$ is in the following form:

(i) $D=\boldsymbol{R}: n$ is an even integer,

$$
T=J_{n / 2}=\left(\begin{array}{cc}
0 & 1_{n / 2} \\
-1_{n / 2} & 0
\end{array}\right),
$$

(ii) $D=C:(p, q)$ is a pair of non-negative integers such that $p+q=n$,

(iii) $D=\boldsymbol{H}$ :

$$
T=-i 1_{p q}=\left(\begin{array}{cc}
-i 1_{p} & 0 \\
0 & i 1_{q}
\end{array}\right),
$$

$$
T=j 1_{n} .
$$

Hence the corresponding special unitary groups $S U_{n}(D, h)$ are given by the following matrix groups:

(i ) $\quad D=\boldsymbol{R}: n$ is even,

$$
S U_{n}(\boldsymbol{R}, h)=S p_{n / 2}(\boldsymbol{R})=\left\{\left.g \in S L_{n}(\boldsymbol{R})\right|^{t} g J_{n / 2} g=J_{n / 2}\right\},
$$

(ii) $\quad D=C: p+q=n$,

$$
S U_{n}(\boldsymbol{C}, h)=S U(p, q, \boldsymbol{C})=\left\{g \in S L_{n}(\boldsymbol{C}) \mid t \bar{g} 1_{p q} g=1_{p q}\right\},
$$

(iii) $^{\prime} \quad D=\boldsymbol{H}$ :

$$
S U_{n}(\boldsymbol{H}, h)=S U_{n}(\boldsymbol{H})^{-}=\left\{g \in S L_{n}(\boldsymbol{H}) i^{t} g^{\iota}\left(j 1_{n}\right) g=\jmath 1_{n}\right\} .
$$


These groups are $\boldsymbol{R}$-algebraic groups, which are of non-compact hermitian type unless $G=S U(n, 0, C) \cong S U(0, n, C) \cong S U(n, C)$ or $S U_{1}(\boldsymbol{H})^{-}$. Moreover these groups are $\boldsymbol{R}$-simple except for the case where $G=S U_{2}(\boldsymbol{H})^{-}$(see (4.12), or [S1], Appendix, §1).

These groups act on bounded symmetric domains as follows. Consider the following set of complex structures on $V$

$$
\begin{aligned}
& \mathscr{D}(V, h) \\
& =\left\{I \in \operatorname{End}_{\boldsymbol{R}}(V) \mid I^{2}=-1_{V}, h(x, I y) \text { is a positive-definite } D \text {-hermitian }\right\} .
\end{aligned}
$$

Then the special unitary group $S U_{n}(D, h)$ acts on $\mathscr{D}(V, h)$ transitively, and $\mathscr{D}(V, h)$ becomes an irreducible hermitian symmetric domain and is isomorphic to a homogeneous space $S U_{n}(D, h) / K$ where $K$ is a maximal compact subgroup of $S U_{n}(D, h)$. A bounded symmetric domain $\mathscr{D}(V, h)$ obtained as above is called a classical domain and isomorphic to one of the following bounded symmetric domains.

$$
\begin{gathered}
(I)_{p q}=\left\{Z \in M(p, q, \boldsymbol{C}) \mid 1_{q}-{ }^{t} \bar{Z} Z \gg 0\right\}, \\
(I I)_{n}=\left\{Z \in M_{n}(\boldsymbol{C}) \mid{ }^{t} Z=-Z, 1_{n}-{ }^{t} \bar{Z} Z \gg 0\right\}, \\
(I I I)_{m}=\left\{\left.Z \in M_{m}(\boldsymbol{C})\right|^{t} Z=Z, 1_{m}-{ }^{t} \bar{Z} Z \gg 0\right\} .
\end{gathered}
$$

The relations between $S U(V, h)$ and $\mathscr{D}(V, h)$ and the $\boldsymbol{R}$-rank of $\operatorname{SU}(V, h)$ are shown in the following table.

$$
\begin{array}{ccccc}
D & G=S U(V, h) & \mathscr{D}=\mathscr{D}(V, h) & \operatorname{dim}_{C} \mathscr{D} & \boldsymbol{R} \text {-rank } \\
\boldsymbol{R} & S p_{n / 2}(\boldsymbol{R}) & (I I I)_{n / 2} & (n / 2)(n / 2+1) / 2 & n / 2 \\
\boldsymbol{C} & S U(p, q, \boldsymbol{C}) & (I)_{p q} & p \cdot q & \min (p, q) \\
\boldsymbol{H} & S U_{n}(\boldsymbol{H})^{-} & (I I)_{n} & n(n-1) / 2 & {[n / 2]}
\end{array}
$$

\section{(3.16) Satake's classification}

A $\boldsymbol{Q}$-symplectic representation $\left(W_{\boldsymbol{Q}}, \rho_{\boldsymbol{Q}}, A_{\boldsymbol{Q}}, I\right)$ of a $\boldsymbol{Q}$-hermitian pair $\left(G_{\boldsymbol{Q}}, H_{0}\right)$ (cf. (1.1)) is called $\boldsymbol{Q}$-primary if $\left(W_{\boldsymbol{Q}}, \rho_{\boldsymbol{Q}}\right)$ is a sum of $G_{\boldsymbol{Q}^{-}}$-stable subspaces isomorphic to an irreducible $\boldsymbol{Q}$-representation $\rho_{1}: G_{\boldsymbol{Q}} \rightarrow G L(V / \boldsymbol{Q})$.

In this section, we review the classification of $\boldsymbol{Q}$-primary standard symplectic representations. In order to classify $\boldsymbol{Q}$-primary symplectic representations, the following proposition is fundamental. For a proof, see [S1, Ch. IV].

(3.17) Proposition. Let $\left(W_{\boldsymbol{Q}}, \boldsymbol{\rho}_{\boldsymbol{Q}}, A_{\boldsymbol{Q}}, I\right)$ be a $\boldsymbol{Q}$-primary symplectic representation of a Q-hermitian pair $\left(G_{Q}, H_{0}\right)$, and $\rho: G_{Q} \rightarrow G L(V)$ an irreducible representation containing in $\left(W_{Q}, \rho_{Q}\right)$. Setting 


$$
D=\operatorname{End}_{G_{Q}}(V), \quad F_{1}=\operatorname{Cent} D, \quad U=\operatorname{Hom}_{G_{Q}}\left(V, W_{Q}\right),
$$

we have the following.

(i) $D$ is a division algebra over $\boldsymbol{Q}$, and $V$ (resp. $U$ ) becomes a left $D$ module (resp. a right D-module).

(ii) There exists a canonical isomorphism

$$
W_{Q} \cong U \otimes_{D} V \text {. }
$$

(iii) There exist a natural involution c on $D, a(D, \varepsilon)$-hermitian form $h$ on $V$ and $a(D,-\varepsilon)$-hermitian form $h^{\prime}$ on $U$ with respect to the involution c such that

$$
A_{Q}=\operatorname{tr}_{D / Q}\left(h^{\prime} \otimes h\right) \text {. }
$$

(iv) The form $h$ on $V$ is $G_{Q}$-invariant. In particular, $\rho$ is reduced to a natural representation over $F_{1}$

$$
\rho_{1}: G_{Q} \longrightarrow S U(V, h)
$$

(with $\left.\operatorname{End}_{G_{Q}}(V)=D\right)$.

(3.21) Definition. A $\boldsymbol{Q}$-primary representation $\left(W_{\boldsymbol{Q}}, \rho_{\boldsymbol{Q}}, A_{\boldsymbol{Q}}, I\right)$ of a $\boldsymbol{Q}$ hermitian pair $\left(G_{Q}, H_{0}\right)$ is said to be standard if $G_{Q}=R_{F_{1} / Q}(S U(V, h))$ and $\rho$ in (3.20) is induced by the universal homomorphism of the scalar restriction (cf. $[\mathrm{W}, 1.13])$.

(3.22) Remark. Satake [S2] determined all $\boldsymbol{Q}$-primary symplectic representation under an reasonable additional condition. Besides the standard one, there exist few nonstandard representations involving skew-symmetric representations and spin representations. But there exist also a $\boldsymbol{Q}$-primary symplectic representation which does not satisfy his condition (see [S1, p 195] for references). In this paper, we will not deal with non-standard case.

A standard representation is determined only by the data $D$, c, $V, U, h, h^{\prime}$ in proposition (3.17). First we have the following.

(3.23) Proposition. ([S1, Ch. IV, §6]). Let $\left(W_{\boldsymbol{Q}}, \rho_{\boldsymbol{Q}}, A_{\boldsymbol{Q}}, I\right)$ be a Q-primary symplectic representation (not necessarily standard) of a $\mathbf{Q}$-hermitian pair $\left(G_{\boldsymbol{Q}}, H_{0}\right)$, and $D, F_{1}, \iota, V, h, U, h^{\prime}$ be as in Lemma (3.17). Then one of the following cases occurs.

(R1) $D=F_{1}$ is a totally real algebraic number field and $\iota=i d e n t i t y$, and $h$ is a symplectic form on $V(\varepsilon=-1)$.

$(\boldsymbol{R} 2, \boldsymbol{\varepsilon}) \quad D$ is a quaternion algebra over a totally real algebraic number field $F_{1}$ and $c$ is the standard involution, $h$ is $a(D, \varepsilon)$-hermitian form $V$ with respect to $\iota$, where $\varepsilon= \pm 1$.

(C) $F_{1}$ is a CM field, i.e. a purely imaginary quadratic extension of a 
totally real algebraic number field $F_{10}, D$ is a central division algebra over $F_{1}, 6$ is an involution of $D$ of the second kind, and $h$ is $a(D, \varepsilon)$-hermitian form with respect to 6 where $\varepsilon= \pm 1$.

Let $D, F_{1}, \iota$ be as in Proposition (3.23). If we set $F_{1}^{+}=\left\{z \in F_{1} \mid z^{\imath}=z\right\}$, then $F_{1}^{+}$is a totally real algebraic number field. Setting $t=\left[F_{1}^{+}: \boldsymbol{Q}\right]$, let $\left\{\tau_{i}: F_{1}^{+} \subset \rightarrow\right.$ $\boldsymbol{R}, 1 \leqq i \leqq t\}$ be the set of $t$-distinct embeddings of $F_{1}^{+}$into $\boldsymbol{R}$. For each: $\tau_{i}$ : $F_{1}^{+} \subset \boldsymbol{R}$, we put

$$
\begin{aligned}
& F_{1}^{(i)}=F_{1} \bigotimes_{F_{1}^{+}, \tau_{i}} R, \\
& D^{\tau_{i}}=D \bigotimes_{F_{1}^{+}, \tau_{i}} \boldsymbol{R}, \\
& W^{\tau_{i}}=W_{Q} \bigotimes_{F_{1}^{+}, \tau_{i}} \boldsymbol{R}, \\
& V^{\tau_{i}}=V \bigotimes_{F_{1}^{+}, \tau_{i}} \boldsymbol{R}, \\
& U^{\tau_{i}}=U \bigotimes_{F_{1}^{+}, \tau_{i}} \boldsymbol{R} .
\end{aligned}
$$

The algebra $D^{\tau_{2}}$ becomes a central simple algebra over $F_{1}^{(i)}$, so there exists a division algebra $D^{(i)}$ over $F_{1}^{(i)}$ such that

$$
D^{\tau_{i}} \cong M_{s}\left(D^{(i)}\right) \text {. }
$$

Fixing an above isomorphism, we denote by $\varepsilon_{\nu \mu}^{i}$ the corresponding matrix unit in $D^{\tau_{i}}$. We moreover set:

$$
V^{(i)}:=\varepsilon_{11}^{i} V^{\tau_{\imath}}, \quad U^{(i)}=U^{\tau_{\imath}} \varepsilon_{11}^{\imath} .
$$

Then $V^{(i)}$ (resp. $U^{(\imath)}$ ) are left (resp. right) $D^{(\imath)}$-modules and we have an isomorphism (cf. [S1], p 189),

$$
W^{\tau_{\imath}}=U^{(i)} \otimes_{D^{(i)}} V^{(\imath)} .
$$

Note that from (3.23), $F_{1}^{(\imath)}$ is isomorphic to $\boldsymbol{R}$ or $\boldsymbol{C}$, corresponding to the case $(\boldsymbol{R} 1),(\boldsymbol{R} 2, \varepsilon)$ or $(\boldsymbol{C})$, so $D^{(i)}$ is isomorphic to $\boldsymbol{R}, \boldsymbol{H}$, or $\boldsymbol{C}$.

Under these notations, we can state the following theorem.

(3.31) Theorem. ([S1, Ch. IV, §6]). Let $\left(W_{\boldsymbol{Q}}, \rho_{\boldsymbol{Q}}, A_{\boldsymbol{Q}}, I\right)$ be a standard $\boldsymbol{Q}$ primary symplectic representation, and $D, \iota, F_{1}, V, h, U, h^{\prime}, W_{Q}=U \otimes_{D} V, A_{Q}=$ $\operatorname{tr}_{D / Q}\left(h^{\prime} \otimes h\right)$ be as in (3.17). Then we have the following.

(i) There exists a decomposition

$$
W_{\boldsymbol{R}}:=W_{Q} \otimes_{Q} R=\bigoplus_{i=1}^{t} W^{i} \cong \bigoplus_{i=1}^{t} U^{(i)} \otimes_{D^{(i)}} V^{(i)} .
$$

(ii) For each $i, 1 \leqq i \leqq t, h$ (resp. $h^{\prime}$ ) induces $a\left(D^{(\imath)}, \varepsilon \eta_{i}\right)$-hermitian form $h^{(i)}$ on $V^{(i)}$ (resp. $\left(D^{(i)},-\varepsilon \eta_{i}\right)$-hermitian form $h^{(i)}$ on $\left.U^{(i)}\right)$, where $\eta_{i}= \pm 1$. We have a decomposition of $A_{\boldsymbol{R}}:=A_{\boldsymbol{Q}} \otimes \boldsymbol{R}=\bigoplus_{i=1}^{t} A^{(\imath)}$ corresponding to (3.32), where one set 


$$
A^{(i)}:=\operatorname{tr}_{D^{(i) / F}}(i)\left(h^{\prime(i)} \otimes h^{(i)}\right) .
$$

(iii) The $\boldsymbol{R}$-valued points $G_{\boldsymbol{R}}$ of $G_{\boldsymbol{Q}}=R_{F_{1} / \boldsymbol{Q}}(S U(V, h))$ has a canonical decomposition

$$
G_{R}=R_{F_{1} / Q}(S U(V, h))_{R}=\prod_{i=1}^{t} S U\left(V^{(i)}, h^{(i)}\right),
$$

and, for each $i$, the natural representation $\rho_{1}: G_{Q} \rightarrow S U(V, h)$ induces a representation

$$
\rho_{1}^{(\imath)}: G_{R}=R_{F_{1} / Q}(S U(V, h))_{R} \longrightarrow S U\left(V^{(\imath)}, h^{(\imath)}\right),
$$

where $\rho_{1}^{(i)}$ can be written in the form

$$
\rho_{1}^{(i)}=1 \otimes \cdots 1 \otimes i d_{V^{(i)}} \otimes 1 \cdots \otimes 1
$$

according to the decomposition (3.34).

Moreover, for each case in (3.23), we have the following

(3.37) Theorem. ([S1, Ch. IV, § 6]). Under the notation in Proposition (3.23), we have the following explicit descriptions of $F_{1}^{(i)}, D^{i}, D^{(i)}, V^{(i)}, h^{(i)}, U^{(i)}, G_{\boldsymbol{R}}$ for the cases of $(\boldsymbol{R} \mathbf{1}),(\boldsymbol{Q} \mathbf{2}, \boldsymbol{\varepsilon}),(\boldsymbol{C})$ respectively.

(R1) $(\varepsilon=-1) D=F_{1}=F_{1}^{+} . \quad$ Set $\operatorname{dim}_{F_{1}} V=n, \operatorname{dim}_{F_{1}} U=m$. Then one has:

$$
F^{(\imath)} \cong D^{\tau_{i}} \cong D^{(i)} \cong \boldsymbol{R}, \quad V^{(\imath)} \cong \boldsymbol{R}^{n}, \quad U^{(i)} \cong \boldsymbol{R}^{m},
$$

$h^{(2)}: \boldsymbol{R}$-symplectic form on $V^{(i)}, \quad\left(\eta_{\imath}=1\right)$ for $1 \leqq i \leqq t=d$,

$$
G_{\boldsymbol{R}} \cong \underbrace{S p_{n / 2}(\boldsymbol{R}) \times \cdots \times S p_{n / 2}(\boldsymbol{R})}_{d} .
$$

$(\boldsymbol{R} 2, \varepsilon)$ We have $F_{1}=F_{1}^{+}$, and $D$ is a quaternion algebra over $F_{1}$. Set $\operatorname{rank}_{D} V=n, \operatorname{rank}_{D} U=m$. Then one has $F^{(\imath)}=\boldsymbol{R}$. After a suitable renumbering of $\left\{\tau_{i}\right\}$, we may assume that for some $t^{\prime}, 0 \leqq t^{\prime} \leqq t$.

$$
D^{\tau_{i}} \cong\left\{\begin{array} { l l } 
{ \boldsymbol { H } } & { 1 \leqq i \leqq t ^ { \prime } } \\
{ M _ { 2 } ( \boldsymbol { R } ) } & { t ^ { \prime } + 1 \leqq i \leqq t , }
\end{array} \quad D ^ { ( i ) } \cong \left\{\begin{array}{ll}
\boldsymbol{H} & 1 \leqq i \leqq t^{\prime} \\
\boldsymbol{R} & t^{\prime}+1 \leqq i \leqq t
\end{array}\right.\right.
$$

Then one has:

$(\varepsilon=1)$

$$
V^{(i)} \cong\left\{\begin{array} { l } 
{ \boldsymbol { H } ^ { n } } \\
{ \boldsymbol { R } ^ { 2 n } , }
\end{array} \quad U ^ { ( i ) } \cong \left\{\begin{array} { l } 
{ \boldsymbol { H } ^ { m } } \\
{ \boldsymbol { R } ^ { 2 m } , }
\end{array} \quad W ^ { \tau _ { i } } \cong \left\{\begin{array}{ll}
\boldsymbol{H}^{n} \bigotimes_{\boldsymbol{H}} \boldsymbol{H}^{m} & 1 \leqq i \leqq t^{\prime} \\
\boldsymbol{R}^{2 n} \bigotimes_{\boldsymbol{R}} \boldsymbol{R}^{2 m} & t^{\prime}+1 \leqq i \leqq t
\end{array}\right.\right.\right.
$$

$$
\begin{aligned}
& h^{(i)}= \begin{cases}\text { positive-definite } \boldsymbol{H} \text {-symmetric form }\left(\eta_{i}=1\right) & 1 \leqq i \leqq t^{\prime}, \\
\boldsymbol{R} \text {-symplectic form }\left(\eta_{i}=-1\right) & t^{\prime}+1 \leqq i \leqq t,\end{cases} \\
& G_{\boldsymbol{R}}=\underbrace{S U_{n}(\boldsymbol{H}) \times \cdots \times S U_{n}(\boldsymbol{H})}_{t^{\prime} \times \text { compact }} \times \underbrace{S p_{n}(\boldsymbol{R}) \times \cdots \times S p_{n}(\boldsymbol{R})}_{\left(t-t^{\prime}\right) \times(\mathrm{III})_{n}} .
\end{aligned}
$$


$(\varepsilon=-1)$

$$
h^{(i)}= \begin{cases}\boldsymbol{H} \text {-symplectic form }\left(\boldsymbol{\eta}_{i}=1\right) & 1 \leqq i \leqq t^{\prime}, \\ \text { positive-definite } \boldsymbol{R} \text {-symmetric form }\left(\eta_{i}=-1\right) & t^{\prime}+1 \leqq i \leqq t,\end{cases}
$$

$$
G_{R}=\underbrace{S U_{n}(\boldsymbol{H})^{-} \times \cdots \times S U_{n}(\boldsymbol{H})^{-}}_{t^{\prime} \times(\mathrm{II})} \times \underbrace{S O_{2 n}(\boldsymbol{R}) \times \cdots \times S O_{2 n}(\boldsymbol{R})}_{\left(t-t^{\prime}\right) \times \text { compact }} .
$$

(C) $(\varepsilon= \pm 1) . \quad F_{1}$ is a purely imaginary quadratic extension of $F_{1}^{+}$, so $t=$ $(1 / 2)\left[F_{1}: \boldsymbol{Q}\right]$. We set $\left[D: F_{1}\right]=r^{2}, \operatorname{rank}_{D} V=n$, and $\operatorname{rank}_{D} U=m$. Then one has:

$$
\begin{gathered}
F_{1}^{(i)} \cong D^{(i)} \cong \boldsymbol{C}, \quad D^{\tau_{i}} \cong M_{r}(\boldsymbol{C}), \\
V^{(i)} \cong C^{n r}, \quad U^{(\imath)} \cong C^{m r}, \quad W^{\tau_{\imath}} \cong \boldsymbol{C}^{m r} \otimes_{C} \boldsymbol{C}^{n r} .
\end{gathered}
$$

We may assume that for $t^{\prime}, 0 \leqq t^{\prime} \leqq t$,

$$
\begin{aligned}
& h^{(\imath)}= \begin{cases}\boldsymbol{C} \text {-symplectic form with the signature }\left(p_{i}, q_{\imath}\right) & 1 \leqq i \leqq t^{\prime}\left(p_{i} \geqq q_{i}\right), \\
\text { positive-definite } \boldsymbol{C} \text {-hermitian form } & t^{\prime}+1 \leqq i \leqq t,\end{cases} \\
& G_{\boldsymbol{R}} \cong \prod_{i=1}^{t^{\prime}} \underbrace{S U\left(p_{i}, q_{i}, \boldsymbol{C}\right)}_{\text {(I) } p_{p q_{i}}} \times \underbrace{S U_{n r}(\boldsymbol{C}) \times \cdots \times S U_{n r}(\boldsymbol{C})}_{\left(t-t^{\prime}\right) \times \operatorname{compact}} .
\end{aligned}
$$

(3.42) Proposition. A Q-algebraic group $G_{Q}=R_{F_{1} / Q}(S U(V, h))$ in (3.37) is Zariski connected. Assume that $G_{\boldsymbol{R}}$ is non-compact, i.e., $\operatorname{dim} \mathscr{D} \geqq 1$. Then $G_{Q}$ is $\boldsymbol{Q}$-simple except for the case $(\boldsymbol{R 2},-\mathbf{1}), \boldsymbol{n}=\mathbf{2}$.

Proof. See [S1, Appendix, §1].

\section{$\S 4$. Vanishing Theorem and the Case $\operatorname{rank}_{R} G_{R} \geqq 2$}

Let $G$ be a connected semi-simple real Lie group with finite center of hermitian type, $K$ a maximal compact subgroup of $G$, so that a quotient space $\mathscr{D}=G / K$ becomes a hermitian symmetric bounded domain. Let $\Gamma$ be a discrete subgroup of $G$ of a finite covolume with respect to the Haar measure. If $\Gamma$ is torsion-free, the quotient space $M=\Gamma \backslash \mathscr{D}$ becomes a smooth quasi-projective variety. For a finite dimensional complex representation $\rho: G \rightarrow G L\left(W_{C}\right)$, we denote by $\boldsymbol{W}_{C}$ the associated local system on $M=\Gamma \backslash \mathscr{D}$. Let $L_{(2)}\left(M, W_{C}\right)$ be as in (2.9), and $H_{(2)}^{\cdot}\left(M, W_{C}\right)$ the $L_{2}$-cohomology group for it. Let $L_{2}(\Gamma \backslash \mathscr{D})^{\infty}$ denote the set of $C^{\infty}$ square-integrable function on $\Gamma \backslash \mathscr{D}$, and view it as a unitary $G$-module under the right translation. Since it is a $(\mathfrak{g}, K)$-module, we may consider the relative Lie algebra complex $C^{*}\left(\mathrm{~g}, K ; L_{2}(\Gamma \backslash G)^{\infty} \otimes W_{C}\right)$, whose cohomology yields the relative Lie algebra cohomology (cf. [B-W]).

First, we recall the following.

(4.1) Theorem. ([B], $[\mathrm{B}-\mathrm{C}])$. There exists a quasi-isomorphism

$$
C^{*}\left(\mathfrak{g}, K ; L_{2}(\Gamma \backslash G)^{\infty} \otimes W_{C}\right) \longrightarrow L_{(2)}^{*}\left(M, W_{C}\right)^{\infty} .
$$


In particular, we have isomorphisms

$$
E x t_{(\mathrm{g}, K)}^{\cdot}\left(W^{*}, L_{2}(\Gamma \backslash G)^{\infty}\right) \cong H_{(2)}^{\cdot}(\Gamma \backslash \mathscr{D}, \mathbb{W}) .
$$

Write $L_{2}(\Gamma \backslash G)^{\infty}$ as the direct sum of the discrete spectrum $L_{2}(\Gamma \backslash G)_{d}^{\infty}$ and its orthogonal complement, the so-called continuous spectrum $L_{2}(\Gamma \backslash G)_{c t}^{\infty}$ 。

The following theorem is a special case of results in [B-C].

(4.2) Theorem. (see [B-C, Prop. 4.4 and Th. 4.5]) Under the assumption as above, we have the following.

(i) $H_{(2)}\left(M, W_{C}\right)$ is finite dimensional, ${ }^{1}$

(ii) there exists a finite set $\left(H_{i}\right),(i \in S)$ of mutually orthogonal closed irreducible G-invariant subspaces of $L_{2}(\Gamma \backslash G)_{d}$ such that

$$
H_{(2)}\left(M, W_{c}\right)=E x t_{(\mathrm{g}, K}^{\cdot}\left(W_{\boldsymbol{c}}^{*}, L_{2}(\Gamma \backslash G)_{d}^{\infty}\right)=\bigoplus_{i \in S} E x t_{(\mathrm{g}, K)}\left(W_{\boldsymbol{c}}^{*}, H_{i}\right),
$$

(4.4) Definition. Let $G$ be as above. We say that $G$ has no compact factor if it has no infinite normal compact subgroup. A discrete subgroup $\Gamma$ of $G$ is said to be irreducible if the image of $\Gamma$ under any surjective morphism $G \rightarrow G^{\prime}$ with non-trivial image and non-compact kernel is non-discrete.

We can prove the following vanishing theorem of $L_{2}$-cohomology group.

(4.5) Theorem. Let $G$ be as above. Assume that $G$ has no compact factor and $\Gamma$ is an irreducible discrete subgroup of $G$ with a finite covolume. If ( $\rho$, $\left.W_{c}\right)$ is a non-trivial finite complex representation of $G$, we have

$$
H_{(2)}^{q}\left(\Gamma \backslash \mathscr{D}, W_{C}\right)=0 \quad \text { for } \quad q<\operatorname{rank}_{R} G,
$$

where $\operatorname{rank}_{R} G$ denote the $R$-rank of $G$.

Proof. If $\Gamma$ is cocompact, then this is nothing but [B-W, Ch. VII, Proposition 6.4]. Thanks to (4.3), their proof works even if $\Gamma \backslash G$ is not compact.

\section{(4.6) Vanishing theorem}

Now we apply this theorem for standard $\boldsymbol{Q}$-primary symplectic representations. Let $\left(W_{\boldsymbol{Q}}, \rho_{\boldsymbol{Q}}, A_{\boldsymbol{Q}}, I\right)$ be a standard $\boldsymbol{Q}$-symplectic representation, $D$, ,,$F_{1}$, $V, U, h, h^{\prime}$ as in (3.17), and $G_{Q}=R_{F_{1} / Q}(S U(V, h))$.

We take a lattice $V_{\boldsymbol{Z}}$ in $V$ (see $\S 1$ ), and set $D_{\boldsymbol{Z}}=\left\{m \in D \mid m V_{\boldsymbol{Z}} \subset V_{\boldsymbol{Z}}\right\}$. Then $D_{\boldsymbol{Z}}$ becomes a $\boldsymbol{Z}$-subalgebra of $D$ such that $D_{\boldsymbol{Z}} \otimes_{\boldsymbol{Z}} \boldsymbol{Q} \cong D$, which is called an order of $D$. Taking a $D_{\boldsymbol{Z}}$-right submodule $U_{\boldsymbol{Z}}$ of $U$, we set

$$
W_{Z}=U_{Z} \otimes_{D_{Z}} V_{Z}
$$

\footnotetext{
${ }^{1}$ Of course, this also follows from the Zucker conjecture (2.11)
} 
Then $W_{\boldsymbol{Z}}$ becomes a lattice in $W_{\boldsymbol{Q}}$ and we may assume that $W_{\boldsymbol{Z}}$ satisfies the condition (1.7), i.e., $A_{\boldsymbol{Q}}\left(W_{\boldsymbol{Z}}, W_{\mathbf{Z}}\right) \subset Z$. From definition (1.4) and the above construction, we have an isomorphism of discrete groups

$$
G_{W_{Z}} \cong G_{V_{Z}} \cdot
$$

Take a torsion-free arithmetic subgroup $\Gamma \subset G_{V_{Z}}$.

Let $G_{\boldsymbol{Q}}=R_{F_{1} / \boldsymbol{Q}}(S U(V, h))$ be as above. Then from (3.37) and (3.42), except for the case $(\boldsymbol{R} 2,-\mathbf{1}), \boldsymbol{n}=\mathbf{2}$, we can write

$$
G_{R}=G_{1} \times \cdots \times G_{l} \times U,
$$

where $G_{i}=S U\left(V^{(\imath)}, h^{(\imath)}\right)$ is a $\boldsymbol{R}$-simple non-compact Lie group of hermitian type for $1 \leqq i \leqq l$ and $U$ is a compact group.

(4.9) Proposition. Assume that $(V, h)$ is not in the case $(\boldsymbol{R 2},-\mathbf{1}), \boldsymbol{n}=\mathbf{2}$. For any torsion-free arithmetic subgroup $\Gamma \subset G_{R}$, let $\Gamma^{\prime}$ denote the image of $\Gamma$ under the projection $G_{R} \rightarrow G_{R}^{\prime}=G_{1} \times \cdots \times G_{l}$ (cf. (4.8)). Then $\Gamma^{\prime}$ is an irreducible torsionfree discrete subgroup with finite covolume.

Proof. It is easy to see that $\Gamma^{\prime}$ is a discrete subgroup in $G_{\boldsymbol{R}}^{\prime}$ with finite covolume. Let $\rho_{1}^{(i)}: G_{R} \rightarrow G_{i}=S U\left(V^{(i)}, h^{(i)}\right)$ be the representation in (3.35) for $1 \leqq i \leqq l$. Then from the construction we can see that $\rho_{i \mid r}$ induces an isomorphism $\Gamma \cong \rho_{\imath}(\Gamma)$. By a corollary in [Shz, No. 4], $\Gamma^{\prime}$ is irreducible in $G_{R}^{\prime}$. Since the projection map $\Gamma \rightarrow \Gamma^{\prime}$ is injective, $\Gamma^{\prime}$ is also torsion-free.

Let $K$ be a maximal compact subgroup of $G_{R}=G_{1} \times \cdots \times G_{l} \times U$, and write $K$ as $K_{1} \times \cdots \times K_{l} \times U$, so that the corresponding hermitian symmetric space $\mathscr{D}=$ $G_{R} / K$ has a decomposition as

$$
\mathscr{D}=\mathscr{D}_{1} \times \cdots \times \mathscr{D}_{l},
$$

where $\mathscr{D}_{\imath}=G_{i} / K_{i}$ are irreducible symmetric spaces. We have a natural isomorphism

$$
M:=\Gamma \backslash \mathscr{D} \cong \Gamma^{\prime} \backslash \mathscr{D}
$$

(4.12) Remark. We have an isomorphism $S U_{2}(\boldsymbol{H})^{-} \cong S U(2, \boldsymbol{C}) \times S L_{2}(\boldsymbol{R})$.

Now we state our main theorem in this section.

(4.13) Theorem. Let $\left(W_{\boldsymbol{Q}}, \rho_{\boldsymbol{Q}}, A_{\boldsymbol{Q}}, I\right)$ be a standard $\boldsymbol{Q}$-primary symplectic representation, which is not the case $(\boldsymbol{R 2},-\mathbf{1}), \boldsymbol{n}=\mathbf{2}$, and $(V, h), \Gamma \subset G_{\boldsymbol{R}}$ as above. Asumme that $\operatorname{rank}_{R} G_{R} \geqq 2$. Then we have

$$
H^{q}\left(M, W_{Q}\right)=0, \quad q \leqq 1 .
$$

Even if $\operatorname{rank}_{R} G_{R}=1$, we have $H^{\circ}\left(M, W_{Q}\right)=0$. 
Proof. From (3.17), $W_{Q}$ is a vector space over a field $F_{1}=\operatorname{Cent}(D)$. The field $F_{1}$ is a totally real field, or a CM field (see (3.23)). Set $t=\left[F_{1}: Q\right]$. Let $\left\{\sigma_{i}: F_{1} \subset C_{C} C\right\}_{i=1}^{t}$ denote the set of $t$-distinct embeddings of $F_{1}$ into $C$. For an embedding $\sigma_{i}: F_{1} \subseteq C$, we put

$$
W^{\sigma_{i}}=W_{Q} \otimes_{F_{1}, \sigma_{i}} C, \quad W^{\sigma_{i}}=W_{Q} \otimes_{F_{1}, \sigma_{i}} C .
$$

By the universal coefficient theorem, we have an isomorphism

$$
H^{q}\left(M, W_{\boldsymbol{Q}}\right) \otimes_{F_{1}, \sigma_{i}} C \cong H^{q}\left(M, W^{\sigma_{i}}\right) .
$$

Note that $W^{\sigma_{i}}$ is a local system on $M$ associated to a representation

$$
\left(\rho_{Q}\right)^{(i)}: G_{R} \longrightarrow G L\left(W^{\sigma_{i}} / C\right)
$$

induced by $\rho_{Q}$. From the assumption and (4.9), an arithmetic group $\Gamma \subset G_{R}$ is irreducible, hence from (2.13) we have isomorphisms

$$
H_{(2)}^{q}\left(M, W^{\sigma_{i}}\right) \cong H^{q}\left(M, W^{\sigma_{i}}\right) \quad \text { for } q \leqq 1 .
$$

From (4.16) and (4.18), in order to show (4.14), it suffices to show that

$$
H_{(2)}^{q}\left(M, W^{\sigma} i\right)=0 \quad \text { for } q \leqq 1 .
$$

Recall that we have an isomorphism $W_{Q}=U \otimes_{D} V$ (see (3.17)). Set $U^{\sigma_{i}}:=$ $U \otimes_{F_{1}, \sigma_{2}} C, V^{\sigma_{i}}:=V \otimes_{F_{1}, \sigma_{i}} C$, and $D^{\sigma_{i}}:=D \otimes_{F_{1}, \sigma_{i}} C$. Choosing an isomorphism $D^{\sigma_{i}} \cong M_{s}(\boldsymbol{C})$, let $\varepsilon_{\mu \nu}^{i}$ denote the matrix unit in $D^{\sigma_{i}}$. Then, as in (3.29) and (3.30), setting $U_{C}^{(i)}:=U^{\sigma_{i}} \varepsilon_{11}^{i}, V_{c}^{(i)}:=\varepsilon_{11}^{i} V^{\sigma_{i}}$, we have an isomorphism

$$
W^{\sigma_{i}} \cong U_{c}^{(i)} \otimes_{c} V_{c}^{(i)}
$$

Assume that $F_{1}$ is totally real. Then, the representation $\rho_{1}: G_{Q} \rightarrow S U(V, h)$ induces a representation

$$
\rho_{1 C}^{(\imath)}: G_{R} \longrightarrow S U\left(V_{C}^{(i)}, h_{C}^{(2)}\right)
$$

which is obtained by a scalar extension of (3.35) from $\boldsymbol{R}$ to $\boldsymbol{C}$. Hence, from (3.36), $\rho_{1 c}^{(i)}$ can be written in form

$$
\rho_{1 C}^{(i)}=1 \otimes \cdots 1 \otimes\left(i d_{V}(i)\right)_{c} \otimes 1 \cdots \otimes 1 .
$$

Write $G_{R}=G_{1} \times \cdots \times G_{l} \times U$ as in (4.8) and take $i$ such that $1 \leqq i \leqq l$. Then since $\rho_{1 C}^{(i)}$ is trivial on the compact factor $U$, it descends to a representation of $G_{R}^{\prime}=$ $G_{1} \times \cdots \times G_{l}$. Let $\Gamma^{\prime}$ be as in (4.9). Then we can apply Theorem (4.5) for $G_{R}^{\prime}$. $\rho_{1 C}^{(i)}, V_{c}^{(i)}, \Gamma^{\prime}$ to deduce that

$$
H_{(2)}^{q}\left(M, V_{C}^{(i)}\right)=0 \quad \text { for } \quad q<\operatorname{rank}_{R} G_{R}^{\prime} .
$$

By the assumption that $\operatorname{rank}_{R} G_{R}^{\prime} \geqq 2$, one has

$$
H_{(2)}^{q}\left(M, V_{c}^{(i)}\right)=0 \quad \text { for } \quad q \leqq 1 .
$$


Hence the assertion (4.19) (so (4.14)) follows from this and the following isomorphism.

$$
H_{(2)}^{q}\left(M, W^{\sigma_{i}}\right) \cong U_{c}^{(i)} \otimes_{c} H_{(2)}^{q}\left(M, \boldsymbol{V}^{(i)}\right) \quad(\text { by }(4.20)) .
$$

The proof for the case when $F_{1}$ is a CM field is similar, so we omit it.

(4.22) Remark. Note that we have the isomorphism $S U_{3}(\boldsymbol{H})^{-} \cong S U(3,1, \boldsymbol{C})$.

By virture of Silverberg's criterion (2.7), as a corollary of (4.13), we obtain the following.

(4.23) Theorem. The Mordell-Weil group $M W(\mathscr{X} / M)$ of a Kuga fiber space $f: \mathscr{X} \rightarrow M$ associated to a standard Q-primary symplectic representation is finite whenever $\operatorname{rank}_{R} G_{R} \geqq 2$.

\section{$\S 5 . \quad R$-Rank 1 and $\Gamma$ Cocompact}

(5.1) In this section, we shall deal with the cases where the $R$-rank of $G_{\boldsymbol{R}}$ is 1 and $\Gamma$ is cocompact. For technical reasons, we exclude the case $(\boldsymbol{R} \boldsymbol{2}$, $-1), n=2$.

From the Satake's classification (cf. Theorem (3.37)), the cases where $G_{R}$ has the $R$-rank 1 are listed as follows:

$$
\begin{aligned}
& \text { Case }(\mathbb{R} 2,-1), n=3 \quad G_{\boldsymbol{R}} \cong S U_{3}(\boldsymbol{H})^{-} \times \underbrace{S O_{6}(\boldsymbol{R}) \times \cdots \times S O_{6}(\boldsymbol{R})}_{\text {possibly }=(1)} \text {, } \\
& \text { Case }(\boldsymbol{C}) \quad G_{R} \cong S U(n r-1,1) \times \underbrace{S U_{n r}(\boldsymbol{C}) \times \cdots \times S U_{n r}(\boldsymbol{C}}_{\text {possibly }=\{1\}}) \text {. }
\end{aligned}
$$

and

$$
\operatorname{dim} \mathscr{D}=1 .
$$

In the above case, we can no more expect the vanishing of the $H^{1}\left(M, \boldsymbol{W}_{C}\right)$ in general, though we have the vanishing of $H^{0}\left(M, W_{C}\right)$ (see (4.13)). In fact, in the case (5.3) when $r=1$ and $t \geqq 2$, there is an arithmetic subgroup $\Gamma \subset G_{W_{Z}}$ such that $H^{1}\left(M, W_{C}\right) \neq 0$ (See [B-W, Ch. vil, §5]). Hence we should consider the Hodge decomposition of $H^{1}\left(M, W_{C}\right)$, and appeal to Theorem (2.31). In this section, we always assume that $\Gamma \backslash \mathscr{D}$ is compact. Note that $\Gamma \backslash \mathscr{D}$ is compact whenever $G_{R}$ has a compact factor.

(5.5) Let $\left(W_{Q}, \rho_{Q}, A_{Q}, I\right)$ be a standard $\boldsymbol{Q}$-primary symplectic representaion, $W_{\boldsymbol{Z}} \subset W_{Q}$ a lattice, $\Gamma \subset G_{W_{Z}} \subset G_{\boldsymbol{R}}$ a torsion free arithmetic subgroup. Let $\left(\boldsymbol{W}_{\boldsymbol{Z}}\right.$, $A, \mathscr{F}^{0}$ ) denote the corresponding VPHS over the smooth manifold $\Gamma \backslash \mathscr{D}$ (see (1.10)). The main result in this section is the following.

(5.6) Theorem. Under the notation as above, we have 


$$
H^{1}\left(M, W_{Q}\right)^{0,0}=0
$$

in the cases (5.2), (5.3), and (5.4).

As a corollary of this theorem, we have the following.

(5.8) Corollary. The Mordell-Weil groups of the Kuga fiber spaces associated to a standard $Q$-primary symplectic representation is finite when $\operatorname{rank}_{R} G_{R}=1$ and $\Gamma \backslash \mathscr{D}$ is compact.

Proof. Since we always have $H^{0}\left(M, W_{C}\right)=0$, by (2.31), Theorem (5.6) implies the assertion.

\section{(5.9) A reduction}

We keep the notation in (5.5). Let $F_{1}, D$ be as in (3.17). Denote by $\left\{\sigma_{1}, \cdots, \sigma_{d}\right\}$ the set of all embeddings $F_{1}$ into $C$ where $d=\left[F_{1}: Q\right]$. Considering $W_{Q}$ as a $F_{1}$-vector, we set $W^{\sigma_{i}}=W_{Q} \otimes_{F_{1}, \sigma_{i}} C$ and $W^{\sigma_{i}}=W_{Q} \otimes_{F_{1}, \sigma_{i}} C$. Then we have the decompositions

$$
\begin{gathered}
\boldsymbol{W}_{C}:=\boldsymbol{W}_{\boldsymbol{Q}} \otimes_{\boldsymbol{Q}} \boldsymbol{C}=\bigoplus_{i=1}^{d} \boldsymbol{W}^{\sigma_{i}}, \\
H^{1}\left(M, \boldsymbol{W}_{C}\right)=\bigoplus_{i=1}^{d} H^{1}\left(M, \boldsymbol{W}^{\sigma_{i}}\right) .
\end{gathered}
$$

Let $\nabla: \mathcal{O}_{M}\left(W_{C}\right) \rightarrow \Omega_{M}^{1} \otimes W_{C}$ denote the Gauss-Manin connection on $W_{C}$. From the horizontality, we have the complex

$$
\nabla: \mathscr{F}^{0} \longrightarrow \Omega_{M}^{1} \otimes G r_{\Phi}^{-1}
$$

whose $H^{1}$ is isomorphic to $H^{1}\left(M, W_{C}\right)^{0,0}$ (see $(2.21)$ ). We have the following

(5.13) Lemma. Assume that the Hodge filtration $\Phi^{0}$ and the Gauss-Manin connection $\nabla$ on $W_{C}$ is compatible with the decomposition (5.10). Then if for at least one $\sigma_{2}: F_{1} \subseteq C$

$$
H^{1}\left(M, W^{\sigma i}\right)^{0,0}=0,
$$

we have $H^{1}\left(M, W_{Q}\right)^{0,0}=0$.

Proof. From the construction of the Hodge structure in (2.15), under the assumption, we have the decomposition

such that

$$
H^{1}\left(M, W^{\sigma_{i}}\right)=\bigoplus_{p+q=0} H_{\sigma_{i}}^{p_{i} q}
$$

$$
H^{1}\left(M, W_{C}\right)^{p, q}=\bigoplus_{i=1}^{d} H_{\sigma_{i}}^{p, q} .
$$

Let $\pi_{i}: H^{1}\left(M, \boldsymbol{W}_{\boldsymbol{Q}}\right) \rightarrow H^{1}\left(M, \boldsymbol{W}^{\sigma_{i}}\right)$ be the natural projection map. Then we have

$$
H^{1}\left(M, W_{Q}\right)^{0,0}=\bigcap_{i=1}^{d} \pi_{i}^{-1}\left(H_{\sigma_{i}}^{0,0}\right) .
$$


Since the map $\pi_{i}$ is injective, this implies the assertion.

\section{(5.15) Gauss-Manin complex}

Let $\left(G_{\boldsymbol{Q}}, H_{0}\right)$ be the $\boldsymbol{Q}$-hermitian pair corresponding to the $\boldsymbol{Q}$-symplectic representation in (5.5), and $K$ the maximal compact subgroup of $G_{R}$ corresponding to $H_{0}$. We also denote by $g_{R}, f$ the Lie algebras of $G_{R}$ and $K$ respectively, and by $\mathfrak{p}$ the orthogonal complement of $f$ in $g_{R}$ with respect to the Killing form. Let us set $W_{c}^{ \pm}=W_{C}\left( \pm i, I_{C}\right), \mathfrak{p}^{ \pm}=\mathfrak{p}_{C}\left( \pm i, a d_{p}\left(H_{0}\right)\right)$. Then, by the condition (1.2), the spaces $W_{c}^{ \pm}$and $\mathfrak{p}^{ \pm}$are stable under the action of $K$, hence they become representations of $K$.

For any representation $T$ of $K$, we can define a holomorphic vector bundle, or a locally free sheaf $\mathscr{I}$ on $M=\Gamma \backslash \mathscr{D}$ as in $[Z 2, \S 2]$. In the notation in $\S 1$, the representations $W_{c}^{+}$(resp. $W_{c}^{-}$) defines a Hodge bundle $\mathscr{F}^{0}$ (resp. $G r_{\Phi}^{-1}$ ) and $\mathfrak{p}^{-}$defines the cotangent sheaf $\Omega_{M}^{1}$ on $M$.

We call the natural complex

$$
\nabla: \mathscr{I}^{0} \longrightarrow \Omega_{M}^{1} \otimes G r_{\mathscr{G}}^{-1}
$$

the (first) Gauss-Manin complex. Then the Gauss-Manin complex in this case is induced by the following homomorphism of the representations of $K$ :

$$
W_{c}^{+} \longrightarrow \mathfrak{p}^{-} \otimes W_{\boldsymbol{c}}^{-} .
$$

\section{(5.18) Proof of Theorem (5.6) in the case (5.3)}

In this case, since $F_{1}$ is a $\mathrm{CM}$ field, we can denote by $\left\{\sigma_{1}, \cdots, \sigma_{t}, \bar{\sigma}_{1}, \cdots, \bar{\sigma}_{t}\right\}$ the set of all embeddings of $F_{1}$ into $C$ such that $\sigma_{i \mid F_{1}^{+}}$is an extension of $\tau_{i}$ : $F_{1}^{+} \subset \boldsymbol{R}$. Since $G_{\boldsymbol{R}}=\prod_{i=1}^{t} S U\left(V^{(\imath)}, h^{(\imath)}\right) \cong S U(n r-1,1, \boldsymbol{C}) \times S U_{n r}(\boldsymbol{C}) \times \cdots \times S U_{n r}(\boldsymbol{C})$, $\left(V^{(1)}, h^{(1)}\right)$ is a $C$-vector space with a skew-hermitian form $h^{(1)}$ such that the signature of $i h^{(1)}$ is $(n r-1,1)$. Recalling that the decomposition $W_{R}=\bigoplus_{i=1}^{t} W^{\tau_{i}}=$ $\oplus_{i=1}^{i} U^{(i)} \otimes_{C} V^{(i)}$, we can write the complex structure $I \in \mathscr{D}\left(W_{R}, A_{R}\right)$ as

$$
I=1_{U^{(i)}} \otimes I_{(1)}+\sum_{i=2}^{t} I_{(i)}^{\prime} \otimes 1_{V^{(i)}},
$$

for some $I_{(1)} \in \mathscr{D}\left(V^{(1)}, h^{(1)}\right) \cong(I)_{n r-1,1}$ and $I_{(2)}^{\prime} \in \mathscr{D}\left(U^{(\imath)}, h^{\prime(\imath)}\right)$. (See [S1, Ch. IV] or [S2]). If we set

$$
H_{0}^{\prime}=I_{(1)}-i \frac{n r-1}{n r+1} 1_{V(\imath)}, \quad H_{0}=H_{0}^{\prime}+\sum_{i=2}^{t} 1_{V^{(i)}},
$$

we can check that $I$ and $H_{0}$ satisfy the condition (1.2). The corresponding maximal compact subgroup $K$ in $G_{R}$ can be written in the form $K=K_{1} \times$ $\prod_{i=2}^{t} S U\left(V^{(\imath)}, h^{(\imath)}\right)$ where $K_{1} \subset G_{1}:=S U\left(V^{(1)}, h^{(1)}\right)$ is the maximal compact subgroup corresponding to $H_{0}^{\prime}$.

Let $g_{1}, \mathfrak{f}_{1}$ denote the Lie algebras of $G_{1}, K_{1}$, and $\mathfrak{p}$ the orthogonal complement 
of $f_{1}$ in $g_{1}$. Then we have the decompositions

and an isomorphism

$$
\mathrm{g}_{1}=\mathfrak{f}_{1} \oplus \mathrm{p}, \quad \mathrm{g}_{R}=\mathfrak{f} \oplus \mathfrak{p} .
$$

$$
\mathscr{D}\left(V^{(1)}, h^{(1)}\right) \cong G_{R} / K \cong G_{1} / K_{1}
$$

We have the expression

$$
W^{\sigma_{i}}=U_{\boldsymbol{c}}^{(i)} \otimes_{C} V_{c}^{(i)}
$$

as in (4.20), and in this case, we have the decomposition

$$
V^{(1)} \otimes_{R} C=V_{C}^{(1)} \oplus \overline{V_{C}^{(1)}} .
$$

We may assume that the natural projection $V^{(1)} \rightarrow V_{C}^{(1)}$ becomes a $C$-linear isomorphism. Then if we set $V_{c}^{(1) \pm}=V_{c}^{(1)}\left( \pm i, I_{(1)}\right)$, we have $\operatorname{dim} V_{c}^{(1)+}=n r-1$, $\operatorname{dim} V_{c}^{(1)-}=1$, and $W^{\sigma_{1} \pm}=U_{c}^{(1)} \otimes V_{c}^{(1) \pm}$. From the description as above, the homomorphism (5.17) of representation of $K$ is compatible with decomposition (5.10) and the $\left(\sigma_{1}\right)$-part of the homomorphism is given by

$$
\begin{aligned}
& W^{\sigma_{1},+} \longrightarrow \mathfrak{p}^{-} \otimes W^{\sigma_{1,-}} \\
& \cong U_{\boldsymbol{C}}^{(1)} \otimes\left[V_{\boldsymbol{C}}^{(1)+} \longrightarrow \mathfrak{p}^{-} \otimes V_{C}^{(1)-}\right] .
\end{aligned}
$$

(5.20) Lemma. The homomorphism (5.19) of the representations of $K$ and $K_{1}$ is an isomorphism.

Proof. It suffices to show that $V_{C}^{(1)+} \rightarrow \mathfrak{p}^{-} \otimes V_{C}^{(1)-}$ is an isomorphism of $K_{1-}$ modules. Since $V_{c}^{(1)+}$ and $\mathfrak{p}^{-} \otimes V_{c}^{(1)-}$ are irreducible representations of $K_{1}$ of dimension $n r-1$ and the homomorphism is not trivial, it must be an isomorphism. ${ }^{2}$

The following corollary shows Theorem (5.6) for the case (5.3).

(5.21) Corollary. In case (5.3), we have

$$
H^{1}\left(M, W^{\sigma_{1}}\right)^{0,0}=0,
$$

so in particular $H^{1}\left(M, \mathbb{W}_{Q}\right)^{0,0}=0$.

Proof. Let $\nabla_{\sigma_{i}}$ denote the Gauss-Manin connection restricted to $\mathbb{W}^{\sigma_{i}}$. Then the corresponding Gauss-Manin complex

$$
\nabla_{\sigma_{1}}: \mathscr{I}_{\sigma_{1}}^{-1} \longrightarrow \Omega_{M}^{1} \otimes G r_{\sigma_{1}}^{-1}
$$

is induced by the homomorphism (5.19). Then by (5.20), this $\nabla_{\sigma_{1}}$ becomes an

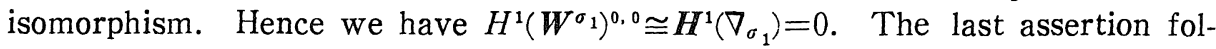
lows from this and Lemma (5.13).

${ }^{2}$ Considering the Harish-Chandra embedding $(I)_{n r-1,1} \subset_{\rightarrow} \mathbb{P}_{C}^{n r-1}$, we can easily see that $\mathfrak{p}^{-} \cong V_{\boldsymbol{C}}^{(1)+} \otimes\left(V_{\boldsymbol{C}}^{(1)-}\right)^{*}$. 
(5.22) Remark. If $t \geqq 2$ and $H^{1}\left(M, \boldsymbol{W}_{C}\right) \neq 0$, we can show that $H^{1}\left(M, W^{\sigma_{i}}\right)^{0,0}$ $\neq 0$ for $i \geqq 2$. Therefore from the example with non-vanishing $H^{1}\left(M, W_{c}\right)$ mentioned in (5.1), we have examples with non-vanishing $H^{1}\left(M, \boldsymbol{W}_{C}\right)^{0,0}$, but still we have (5.21).

\section{(5.23) Proof of Theorem (5.6) in the case of (5.2)}

In this case, $F_{1}$ is a totally real field, and $D$ is a quaternion algebra over $F_{1}$. We denote by $\sigma_{i}: F_{1} \leftrightarrows C$ the embedding which is the extension of $\tau_{i}$. Since $G_{R}=\prod_{i=1}^{t} S U\left(V^{(i)}, h^{(i)}\right) \cong S U_{3}(\boldsymbol{H}) \times S O_{6}(\boldsymbol{R}) \times \cdots \times S O_{6}(\boldsymbol{R}),\left(V^{(1)}, h^{(1)}\right)$ is a left $\boldsymbol{H}$-module of rank 3 with a $\boldsymbol{H}$-skew-hermitian form $h^{(1)}$. Recall that the expression $W^{\sigma_{1}} \cong U_{C}^{(1)} \otimes_{C} V_{C}^{(1)}$ as in (4.23). Let us take a complex structure $I_{(1)} \in$ $\mathscr{D}\left(V^{(1)}, h^{(1)}\right) \cong(I I)_{3}$ and define $V_{C}^{(1) \pm}=V_{C}^{(1)}\left( \pm i, I_{(1)}\right)$. Then we have the decomposition $V_{C}^{(1)}=V_{C}^{(1)+} \oplus V_{C}^{(1)}$. . Setting $H_{0}=(1 / 2) I_{(1)}+\sum_{i=2}^{t} 1_{V^{(i)}}$, we obtain the associated maximal compact subgroup $K=K_{1} \times \Pi_{i=2}^{t} S U\left(V^{(i)}, h^{(i)}\right)$ of $G_{R}=G_{1} \times$ $\prod_{i=2}^{t} S U\left(V^{(\imath)}, h^{(\imath)}\right)$. Then as in (5.19), we have the homomorphism of representations of $K$ and $K_{1}$ :

$$
\begin{aligned}
& W^{\sigma_{1}+} \longrightarrow \mathfrak{p}^{-} \otimes W^{\sigma_{1},-} \\
& \cong U_{c}^{(1)} \otimes\left[V_{c}^{(1)+} \longrightarrow \mathfrak{p}^{-} \otimes V_{c}^{(1)-}\right]
\end{aligned}
$$

In this case, we have the isomorphism $S U(3,1, \boldsymbol{C}) \cong S U_{3}(\boldsymbol{H})^{-}$, which is induced as follows. Let $(T, h)$ be a complex vector space of dimension 4 with a hermitian form $h$ of signature $(3,1)$, and set $G=S U(T, h) \cong S U(3,1, C)$. Let $I^{\prime} \in$ $\mathscr{D}(T, i h)$, and set $T^{ \pm}=T\left( \pm i, I^{\prime}\right)$. Note that $\operatorname{dim} T^{+}=3$ and $\operatorname{dim} T^{-}=1$. Then the space $\wedge^{2} T$ has a hermitian form $h^{\prime}$ induced by $h$, and the decomposition

$$
\wedge^{2} T=\wedge^{2} T^{+} \oplus\left(T^{+} \otimes T^{-}\right)
$$

corresponds to an element $I^{\prime \prime} \in \mathscr{D}\left(\wedge^{2} T, h^{\prime}\right)$. It is known that $\mathscr{D}\left(\wedge^{2} T, i h^{\prime}\right) \cong(I I)_{3}$ and the correspondence $T^{+} \mapsto \wedge^{2} T^{+}$induces an isomorphism $(I)_{3,1} \cong(I I)_{3}$ (cf. $\S 5$, IV, $[\mathrm{S} 1])$, which can be lifted to a group isomorphism $S U(3,1, \boldsymbol{C}) \cong S U_{3}(\boldsymbol{H})^{-}$. Thus the homomorphism $V_{C}^{(1)+} \rightarrow \mathfrak{p}-\otimes V_{C}^{(1)}-$ in (5.24) is isomorphic to

$$
\wedge^{2} T^{+} \longrightarrow \mathfrak{p}^{-} \otimes\left(T^{+} \otimes T^{-}\right)
$$

as a homomorphism of representation of $K_{1}$ (and $K$ ). Since we have an isomorphism $\mathfrak{p}^{-} \cong T^{+} \otimes\left(T^{-}\right)^{*}$ as $K_{1}$-modules (cf. (5.20)), the homomorphism (5.25) is isomorphic to

$$
\wedge^{2}: \wedge^{2} T^{+} \longrightarrow T^{+} \otimes T^{+} .
$$

Hence it is trivial that the homomorphism $\wedge^{2}$ is injective and

$$
\operatorname{coker}\left(\wedge^{2}\right) \cong S^{2}\left(T^{+}\right) \text {. }
$$

Let $I$ denote the locally free sheaf on $M$ corresponding to the representation $T^{+}$. Then, from (5.24), we have the isomorphism 


$$
\operatorname{coker} \nabla_{\sigma_{1}} \cong U_{C}^{(1)} \otimes S^{2}(\mathscr{I}) \text {. }
$$

Now we have the following result which implies Theorem (5.6) in the case (5.2).

(5.28) Proposition. In the case (5.2), we have

$$
H^{1}\left(M, W^{\sigma_{1}}\right)^{0,0}=0 \text {. }
$$

Proof. Since from (5.27)

$$
\boldsymbol{H}^{1}\left(\nabla_{\sigma_{1}}\right) \cong H^{0}\left(\operatorname{coker}\left(\nabla_{\sigma_{1}}\right)\right) \cong U_{C}^{(1)} \otimes H^{0}\left(M, S^{2}(I)\right),
$$

we only have to show that $H^{0}\left(M, S^{2}(\mathscr{T})\right)=0$. Let $\boldsymbol{T}_{C}$ denote the local system on $M$ induced by $T$. Since we have the natural inclusion $\mathscr{I} \subset \mathcal{O}_{M}\left(T_{C}\right)$, we also have the inclusion

$$
H^{0}\left(M, S^{2}(\mathscr{I})\right) \subset H^{0}\left(M, S^{2}\left(\mathbb{T}_{C}\right)\right) .
$$

Then since the right hand side of (5.29) vanishes by Theorem (4.13), we have the assertion.

\section{(5.30) Proof of Theorem (5.6) in the case (5.4)}

In this case, we always have $G_{\boldsymbol{R}} \cong G_{1} \times K_{2} \times \cdots \times K_{t}$ where $G_{1} \cong S L_{2}(\boldsymbol{R}) \cong$ $S p_{1}(R) \cong S U(1,1)$ and $K_{i}$ are compact. We also have a expression $W^{\sigma_{1}} \cong U_{C}^{(1)} \otimes$ $V_{C}^{(1)}$ where $V_{C}^{(1)}$ is a complex irreducible representation of $S L_{2}(\boldsymbol{R})$ and $U_{C}^{(1)}$ is a trivial representation. Then since $M=\Gamma \backslash \mathscr{D}$ is compact, we can apply the result in $[Z 2,(5.33)$, Example $]$ to deduce that

$$
H^{1}\left(M, W^{\sigma_{1}}\right)^{0,0}=0 .
$$

Hence, as before, we have the assertion.

\section{§6. R-Rank 1 and $\Gamma$ Non-Cocompact}

(6.1) Let $\left(W_{\boldsymbol{Q}}, \rho_{Q}, A_{\boldsymbol{Q}}, I\right)$ be a standard $\boldsymbol{Q}$-symmplectic representation, $W_{\boldsymbol{Z}} \subset$ $W_{Q}$ a $Z$-lattice, $\Gamma\left(\subset G_{W_{Z}} \subset G_{R}\right)$ a torsion free arithmetic group. In this section, we assume that $\operatorname{rank}_{R} G_{R}=1$ and $\Gamma \subset G_{R}$ is not cocompact. Again, we will not deal with the case $(\boldsymbol{R} 2,-\mathbf{1}), \boldsymbol{n}=\mathbf{2}$. If $\operatorname{dim} \mathscr{D}=\mathbf{1}$, we can deduce the finiteness results from Zucker's results in $[Z 1]$ (see Remark (6.30)). Hence we will assume that $\operatorname{dim} \mathscr{D}>1$ unless we state otherwise.

We only have to consider the following cases:

$$
\begin{gathered}
\text { Case }(\boldsymbol{R} 2,-\mathbf{1}), \boldsymbol{n = 3} G_{\boldsymbol{R}} \cong S U_{3}(\boldsymbol{H})^{-} \cong S U(3,1, \boldsymbol{C}), \\
\text { Case }(\boldsymbol{C}) \quad G_{\boldsymbol{R}} \cong S U(n r-1,1, \boldsymbol{C}) .
\end{gathered}
$$

In the above cases, the bounded symmetric domain $\mathscr{D} \cong G_{R} / K$ is isomorphic to 
the m-dimensional unit ball $B^{m} \subset C^{m}$ for some $m \geqq 1$. Since $\Gamma \subset G_{\boldsymbol{R}}$ is a torsion free arithmetic subgroup of $G_{R}, M=\Gamma \backslash \mathscr{D}$ is a smooth complex manifold with a finite invariant measure, but, by assumption, is not compact. The BailyBorel-Satake compactification $M^{*}$ of $M$ can be obtained by adding a finite number of cusps $\left\{p_{i}\right\}$ to $M$. Note that $M^{*}$ is projective. Moreover, according to Hemperly [He], a resolution of singularities $\pi: \bar{M} \rightarrow M^{*}$ is obtained by the blowing up of the cusps $\left\{p_{\imath}\right\}$, and the inverse images $D_{i}=\pi^{-1}\left(p_{i}\right)$ are abelian varieties.

(6.4) Let $\left(W_{\boldsymbol{Q}}, \rho_{Q}, A_{Q}, I\right)$ be a standard $\boldsymbol{Q}$-symplectic representation in the case (6.2) or (6.3), $D, \iota, F_{1}, V, U, h, h^{\prime}$ be as in (3.17). Let $f: \mathscr{X} \rightarrow M$ denote the Kuga fiber space associated to the above representation and the lattice $W_{Z}$ in (6.1). Then, as in (2.29), we have the exact sequence

$$
0 \longrightarrow W_{Z} \longrightarrow G r_{\mathscr{F}}^{-1} \longrightarrow \mathcal{O}_{M}^{a n}(\mathscr{X}) \longrightarrow 0 \text {. }
$$

Let us assume that the local monodromy around each $D_{i}$ is unipotent. This is always possible if one replaces $\Gamma$ with a normal subgroup $\Gamma^{\prime}$ of finite index. Then we can extend the abelian scheme $f: \mathscr{X} \rightarrow M$ to a semi-abelian scheme $\bar{f}: \bar{X} \rightarrow \bar{M}$ as follows. Let $\mathscr{W}:=\mathcal{O}_{M} \otimes W_{C}$. Then we have the Gauss-Manin connection $\nabla: \mathscr{W} \rightarrow \Omega_{M}^{1} \otimes \mathscr{W}$ which is integrable. Let $\overline{\mathscr{W}}$ denote the Deligne canonical extension of $\mathscr{W}$ which is a locally free $\mathcal{O}_{\bar{M}}$-module with a logarithmic connection $\bar{\nabla}: \overline{q \bar{q}} \rightarrow \Omega \frac{1}{M}(\log D) \otimes \overline{q l}$ such that $\operatorname{Res}_{D_{i}}(\nabla)$ is nilpotent (see [D1]). Let 1: $M G \bar{M}$ denote the inclusion. We set:

$$
\bar{\Psi} \bar{T}^{p}:=\jmath * \mathscr{I}^{p} \cap \overline{\mathscr{W}} \text {. }
$$

By the nilpotent orbit theorem $[\mathrm{Sc},(4.12)]$, these are locally free subsheaf of $\overline{\mathscr{Y}}$. As in [Z3], we can obtain a semi-abelian scheme $\bar{f}: \bar{X} \rightarrow \bar{M}$ which is an extension of the original abelian scheme $f$ and fits into the following sheaf exact sequence

$$
0 \longrightarrow j_{*} \boldsymbol{W}_{Z} \longrightarrow G r \bar{g}^{-1} \longrightarrow \mathcal{O} \frac{a^{n}}{\bar{M}}(\overline{\bar{X}}) \longrightarrow 0 .
$$

(6.8) Proposition. Under the notations and the assumptions as above, the natural restriction map (see (2.4))

$$
r: H^{0}\left(\bar{M}, \mathcal{O}_{\bar{M}}^{a}(\bar{X})\right) \longrightarrow H^{0}\left(M, \mathcal{O}_{M}(\mathscr{X})\right) \cong M W(\mathscr{X} / M)
$$

is injective and has a finite cokernel.

Proof. First, I remark that all sections $H^{0}\left(\bar{M}, \mathcal{O}_{\bar{M}}^{a n}(\bar{X})\right)$ is algebraic, so $r$ is well-defined. The injectivity of $r$ is obvious. To prove $r$ has a finite cokernel, we first remark that we can costruct the Néron model $N(f): N(\mathscr{X}) \rightarrow$ $\bar{M}$ of $f: \mathscr{X} \rightarrow M$ which has the following properties.

(i) $N(f): N(\mathscr{X}) \rightarrow \bar{M}$ is a group scheme over $\bar{M}$ which is an extension of $f$. 
(ii) Let $Y \rightarrow \bar{M}$ be a smooth morphism and $\phi: Y \cdots \rightarrow N(\mathscr{X})$ a rational map over $\bar{M}$. Then $\phi$ extends to a morphism $\phi: Y \rightarrow N(\mathscr{X})$.

(iii) The semi-abelian scheme $\bar{X}$ is a connected component of $N(\mathscr{X})$, i.e. $\bar{X}$ is a subgroup scheme of $N(\mathscr{X})$ such that for each closed point $p \in \bar{M}, \bar{X}_{p}$ is the connected component of $N(\mathscr{X})_{p}$ containing the identity.

Moreover there exists a projective manifold $\overline{N(\mathscr{X})}$ containing $N(\mathscr{X})$ as a Zariski open set and a projective morphism $\overline{N(f)}: \overline{N(\mathscr{X})} \rightarrow \bar{M}$ which is an extension of $N(f)$ such that $N(\mathscr{X})$ is the maximal open subset of $\overline{N(\mathscr{X})}$ where $\overline{N(f)}$ is smooth. The existence of the above Néron model $N(\mathscr{X})$ and its projective completion is proved as follows. It suffices to show that the existence of them over a some tubular neighborhood $U$ of an irreducible component $D_{i}$ of $D=\sum_{i=1}^{i} D_{i}$. For each point $p \in D_{i}$, we can take a neighborhood $U_{p}$ which is isomorphic to $\Delta^{n}=\left\{\left(z_{i}\right) \in \mathbb{C}^{n}|| z_{i} \mid<1\right\}$ and $U_{p} \cap D_{i}=\left\{z_{1}=0\right\}$. Then the Néron

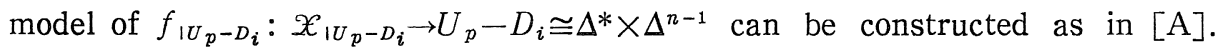
Since the Néron model has a uniqueness property, such local Néron models can be patched together and one gets a global Néron model over the tubular neighborhood $U$ of $D_{i}$.

Now we prove that the finiteness of cokernel of $r$. Every algebraic section $s: M \rightarrow \mathscr{X}$ defines a rational map $\bar{s}: \bar{M} \cdots \rightarrow \overline{N(\mathscr{X})}$. Considering locally around $D$, we can show that $\bar{s}$ must actually map to $N(\mathscr{X})$. Then by the property (ii), $\bar{s}$ is a morphism $\bar{s}: \bar{M} \rightarrow N(\mathscr{X})$ and so it is a section of $N(f)$. This shows that $H^{0}\left(M, \mathcal{O}_{M}(\mathscr{X})\right)$ is isomorphic to $H^{0}\left(\bar{M}, \mathcal{O}_{\bar{M}}(N(\mathscr{X}))\right)$, i. e. the group of sections of $N(f): N(\mathscr{X}) \rightarrow \bar{M}$. Then the cokernel of $r$ is a subgroup of $H^{0}(\bar{M}, N(\mathscr{X}) / \bar{X})$, where $N(\mathscr{X}) / \bar{X}$ is a finite group scheme over $D$. Since the fiber $N(\mathscr{X}) / \bar{X}$ over each component $D_{i}$ is a finite group, $H^{0}(D, N(\mathscr{X}) / \bar{X})$ is also a finite group, and this completes the proof.

\section{(6.9) Hodge theory for $j_{*} W_{C}$}

Let $\left(\boldsymbol{W}_{\boldsymbol{Z}}, \mathcal{A}, \mathscr{F}^{*}\right)$ be the VPHS (see (1.10)) over $\Gamma \backslash \mathscr{D}$ of weight -1 associated to the symmplectic representation as in (6.4). As in (6.1), there exists a projective manifold $\bar{M}$ and an inclusion $\jmath: M \subset \bar{M}$ such that $D=\bar{M}-M$ is a union of smooth hypersurfaces each of which is isomorphic to an abelian variety.

It is known that the cohomology group $H^{i}\left(\bar{M}, j_{*} W_{\boldsymbol{Z}}\right)$ has a polarized pure Hodge structure of weight $i-1$. This fact can be considered as a generalization of Zucker's results in [Z1] to the cases of the higher dimensional bases, and was proved by Cattani-Kaplan-Schmid [C-K-S] and Kashiwara-Kawai [K-K] as follows.

One can see that $M$ admits a complete Kähler metric with Poincaré singularities along $D$. In the above case, $j_{*} W_{C}$ equals the intersection complex $g C \cdot\left(\bar{M}, W_{C}\right)$ of Deligne and Goresky-MacPherson. Then they showed that $9 \mathcal{C} \cdot\left(\bar{M}, W_{C}\right)$ is quasi-isomorphic to the $L_{2}$-complex $\mathcal{L}_{(2)}\left(M, W_{C}\right)$ with respect to 
the above Kähler metric on $M$ and the Hodge metric on $W_{C} \cdot{ }^{3}$ Therefore we have the isomorphisms

$$
H^{i}\left(\bar{M}, j_{*} \boldsymbol{W}_{C}\right) \cong I H^{2}\left(\bar{M}, \boldsymbol{W}_{C}\right) \cong H_{(2)}^{\imath}\left(\Lambda I, \boldsymbol{W}_{C}\right) .
$$

Each element of $L_{2}$-cohomology group can be represented by a harmonic form, so by using the Kähler identity between the Laplacians (cf. [Z1]), we obtain a Hodge decomposition of the cohomology group. (See also [ShzY].)

\section{(6.10) Mixed Hodge theory}

We will recall a more explicit desciption of the Hodge structure on $H^{2}(\bar{M}$, $j_{*} W_{c}$ ) in our case following [ShzY] (cf. [Z1]). In order to see this, we shall introduce the mixed Hodge structure on $H^{\imath}\left(M, W_{Q}\right)$.

Since we have $H^{i}\left(M, \boldsymbol{W}_{\boldsymbol{Q}}\right) \cong H^{i}\left(\bar{M}, \boldsymbol{R} j_{*} \boldsymbol{W}_{\boldsymbol{Q}}\right)$, we have the long exact sequence of cohomology groups

$$
\longrightarrow H^{i}\left(\bar{M}, j_{*} \boldsymbol{W}_{\boldsymbol{Q}}\right) \longrightarrow H^{2}\left(M, \boldsymbol{W}_{\boldsymbol{Q}}\right) \longrightarrow H^{i-1}\left(\bar{M}, R^{1} j_{*} \boldsymbol{W}_{\boldsymbol{Q}}\right) \stackrel{\delta}{\longrightarrow}
$$

which comes from the Leray spectral sequence for the inclusion $j: M \hookrightarrow \bar{M}$. Then it is known that $H^{i}\left(M, W_{Q}\right)$ and $H^{i-1}\left(\bar{M}, R^{1} j_{*} W_{Q}\right)$ has a mixed Hodge structure, which makes (6.11) an exact sequence of mixed Hodge structures.

There are a weight filtration $\{W$.$\} on H^{i}\left(M, W_{Q}\right)$ and the Hodge filtration $\{F \cdot\}$ on $H^{i}\left(M, W_{C}\right)$ such that for each $k, G r_{k}^{W}\left(H^{i}\left(M, W_{Q}\right)\right)$ with the induced Hodge filtration $F^{\cdot}$ forms a polarized (pure) Hodge structure. In our case, we have 3-step weight filtration $0=W_{-1} \subset W_{0} \subset W_{1} \subset W_{2}=H^{\imath}\left(M, W_{Q}\right)$, such that

$$
\begin{gathered}
W_{0}\left(H^{i}\left(M, \boldsymbol{W}_{\boldsymbol{Q}}\right)\right) \cong \operatorname{Im}\left\{H^{i}\left(\bar{M}, j_{*} \boldsymbol{W}_{\boldsymbol{Q}}\right) \longrightarrow H^{i}\left(M, \boldsymbol{W}_{\boldsymbol{Q}}\right)\right\}, \\
G r_{1}^{W} \cong \operatorname{ker}\left\{H^{i-1}\left(D, P_{0}\right) \longrightarrow H^{i+1}\left(\bar{M}, j_{*} \boldsymbol{W}_{\boldsymbol{Q}}\right)\right\}, \\
G r_{2}^{W} \cong \operatorname{ker}\left\{H^{i-1}\left(D, P_{1}\right) \longrightarrow H^{i+1}\left(\bar{M}, j_{*} \boldsymbol{W}_{\boldsymbol{Q}}\right)\right\},
\end{gathered}
$$

where the $P_{k}$ 's denote the local systems on $D$ which underlies VPHS coming from the limit Hodge structure along $D$. (See $[\operatorname{ShzY},(3.1 .4)]$ ).

One can show that there is a quasi-isomorphism $R j_{*} W_{C} \cong \Omega \dot{\bar{M}}(\log D) \otimes \overline{\mathcal{W}}$ (cf. $[\mathrm{ShzY},(3.1 .1)])$. Hence we have an isomorphism $H^{i}\left(M, W_{C}\right) \cong \boldsymbol{H}^{i}(\bar{M}, \Omega \dot{\bar{M}}(\log D)$ $\otimes \overline{\mathscr{W}})$. The Hodge filtration $\left\{F^{\cdot}\right\}$ on the complex $K_{\dot{c}} \cong \Omega \dot{\bar{M}}(\log D) \otimes \bar{\Psi}$ can be defined by

$$
F^{p} K_{\dot{C}}:=\Omega^{i} \bar{M}_{M}(\log D) \otimes \bar{\Psi}^{p-\imath},
$$

and this induces a Hodge filtration on $H^{i}\left(M, \boldsymbol{W}_{C}\right)$. The spectral sequence induced by this filtration

$$
E_{1}^{p, q}=\boldsymbol{H}^{p+q}\left(\bar{M}, G r_{F}^{p} \Omega \dot{\bar{M}}(\log D)\right) \Longrightarrow H^{p+q}\left(M, W_{C}\right)
$$

${ }^{3}$ Actually, they proved this result for the more general case where $\bar{M}-M$ is a divisor with normal crossings. 
degenerates at $E_{1}$.

(6.15) Now we restrict our attention to $H^{1}$. From (6.11), one has the exact sequence of the mixed Hodge structures

$$
0 \longrightarrow H^{1}\left(\bar{M}, j_{*} \boldsymbol{W}_{\boldsymbol{Q}}\right) \longrightarrow H^{1}\left(M, \boldsymbol{W}_{\boldsymbol{Q}}\right) \longrightarrow H^{0}\left(D, R^{1} j_{*} \boldsymbol{W}_{\boldsymbol{Q}}\right) \stackrel{\tilde{o}}{\longrightarrow} .
$$

From (6.13), we have the 3-step Hodge filtration $0=F^{2} \subset F^{1} \subset F^{0} \subset F^{-1}$ on $H^{1}(M$, $W_{C}$ ) whose successive quotients are:

$$
\begin{gathered}
H^{1,-1}=G r_{F}^{1}=F^{1}=\boldsymbol{H}^{1}\left(0 \longrightarrow \Omega \frac{1}{M}(\log D) \otimes \bar{\Phi}^{0} \longrightarrow \Omega_{\bar{M}}^{2}(\log D) \otimes G r_{\bar{\Phi}}^{-1}\right), \\
H^{0,0}=G r_{F}^{0}=F^{0} / F^{1}=\boldsymbol{H}^{1}\left(\bar{\Psi}^{0} \longrightarrow \Omega \frac{1}{\bar{M}}(\log D) \otimes G r_{\bar{\Phi}}^{-1}\right), \\
H^{-1,1}=G r_{F}^{-1}=F^{-1} / F^{0}=\boldsymbol{H}^{1}\left(G r_{\bar{\Phi}}^{-1}\right) .
\end{gathered}
$$

(6.20) Proposition. Let us denote by $H^{1}\left(\bar{M}, j_{*} W_{C}\right)^{p, q}$ the $(p, q)$-component of the pure Hodge structure of $H^{1}\left(\bar{M}, j_{*} W_{C}\right)$. Then we have

(i) the isomorphism

$$
H^{1}\left(\bar{M}, j_{*} W_{C}\right)^{-1,1} \cong H^{1}\left(\bar{M}, G r_{\bar{q}}^{-1}\right),
$$

(ii) and the inclusion

$$
H^{1}\left(\bar{M}, j_{*} \boldsymbol{W}_{\boldsymbol{C}}\right)^{0,0} \subset \boldsymbol{H}^{1}\left(\bar{\Phi}^{0} \longrightarrow \Omega \frac{1}{\bar{M}}(\log D) \otimes G r_{\bar{\Phi}^{-1}}^{-1} .\right.
$$

Proof. These come from (6.18), (6.19) and the fact that (6.16) is an exact sequence of mixed Hodge structures.

(6.21) Now we have the following proposition which is a generalization of (2.27) (cf. [Z1, (10.2)]).

(6.22) Proposition. Let $f: \mathfrak{X} \rightarrow M$ be a Kuga fiber space as in (6.4) and $\bar{f}$ : $\bar{X} \rightarrow \bar{M}$ the extended semi-abelian scheme. Then we have an isomorphism

$$
H^{0}\left(\bar{M}, \mathcal{O}_{\bar{M}}^{a n}(\bar{X})\right) \cong H^{1}\left(\bar{M}, j_{*} \boldsymbol{W}_{\boldsymbol{Z}}\right)^{0,0} .
$$

Here we set $H^{1}\left(\bar{M}, j_{*} \boldsymbol{W}_{\boldsymbol{Z}}\right)^{0,0} \cong i^{-1}\left(H^{0,0}\right)$ where $i: H^{1}\left(\bar{M}, j_{*} \boldsymbol{W}_{\boldsymbol{Z}}\right) \rightarrow H^{1}\left(\bar{M}, j_{*} \boldsymbol{W}_{\boldsymbol{C}}\right)$ is the natural map.

Proof. In this case, $H^{0}\left(\bar{M}, G r_{\bar{g}}^{-1}\right)=0$, because $H^{0}\left(M, W_{C}\right)=0$ by $(2.12)$ and (4.5). Therefore, from (6.7), we have the long exact sequence

$$
0 \longrightarrow H^{0}\left(\bar{M}, \mathcal{O}_{\bar{M}}^{a n}(\bar{X})\right) \longrightarrow H^{1}\left(\bar{M}, j_{*} \boldsymbol{W}_{\boldsymbol{Z}}\right) \stackrel{p}{\longrightarrow} H^{1}\left(\bar{M}, G r_{\overline{\bar{G}}}^{-1}\right) .
$$

which implies that

$$
H^{0}\left(\bar{M}, \mathcal{O}_{\bar{M}}^{a n}(\bar{X})\right) \cong \operatorname{ker}\left\{p: H^{1}\left(\bar{M}, j_{*} \boldsymbol{W}_{\boldsymbol{Z}}\right) \longrightarrow H^{1}\left(\bar{M}, G r_{\bar{q}}^{-1}\right)\right\} .
$$

Since $H^{1}\left(\bar{M}, G r_{\bar{g}}^{-1}\right) \cong H^{-1,1}$ by (6.20), the map $p$ is coincides with the composite of $i$ and the projection from $H^{1}\left(\bar{M}, j_{*} W_{C}\right)$ to its $(-1,1)$-part. Let us take an 
element $u \in \operatorname{ker} p$. Since $u \in H^{1}\left(\bar{M}, j_{*} W_{Z}\right)$ is real and $u$ has no $(-1,1)$-component, it has also no $(1,-1)$-component. Thus $u$ is of type $(0,0)$, and conversely.

(6.23) Corollary. Let $f: \mathscr{X} \rightarrow M$ be a Kuga fiber space as in (6.4). The Mordell-Weil group $M W(\mathscr{X} / M)$ is finite if and only if

$$
H^{1}\left(\bar{M}, j_{*} W_{Q}\right)^{0,0}=0 \text {. }
$$

Proof. By Proposition (6.8), we only have to prove that the group $H^{0}(\bar{M}$, $\left.\mathcal{O}_{\bar{M}}^{a n}(\overline{\mathscr{X}})\right)$ is finite. Since $H^{1}\left(\bar{M}, j_{*} \boldsymbol{W}_{\boldsymbol{Z}}\right)^{0,0} \otimes \boldsymbol{Q} \cong H^{1}\left(\bar{M}, j_{*} \boldsymbol{W}_{\boldsymbol{Q}}\right)^{0,0}$, Proposition (6.22) implies that the condition (6.24) is equivalent to the finiteness of $H^{\circ}\left(\bar{M}, \mathcal{O}_{\bar{n}}(\bar{x})\right)$.

(6.25) Theorem. Let $f: \mathscr{X} \rightarrow M$ be the Kuga fiber spaces associated to the $Q$-symplectic representation of type (6.2) or (6.3). Assume that $M=\Gamma \backslash \mathscr{D}$ is not compact. Then the Mordell-Weil group $M W(\mathfrak{X} / M)$ is finite.

Proof. We first remark that we can replace $M=\Gamma \backslash \mathscr{D}$ with its finite unramified covering. So we may assume that local monodromies around the components of $D$ are unipotent.

We first prove the case (6.3). We shall use the notation in (5.18). In this case, $F_{1}$ is a purely imaginary quadratic field over $\boldsymbol{Q}$, so denote by $\{\sigma, \bar{\sigma}\}$ the embedding of $F_{1}$ into $C$. We have the decomposition

$$
W_{C}=W^{\sigma} \oplus W^{\bar{\sigma}}
$$

where we put $W^{\sigma}:=W_{Q} \otimes_{F_{1}, \sigma} C$. We also have the expression

$$
W^{\sigma}=U_{c} \otimes_{c} V_{c}
$$

where $V_{C}$ is an $n r$-dimensional $\boldsymbol{C}$-vector space which has a $C$-symplectic form $h_{C}$ such that the signature of $\sqrt{-1} h_{C}$ is $(n r-1,1)$. As in (5.18), a complex structure $I \in \mathscr{D}$ defines a decomposition $V_{c}=V_{c}^{+} \oplus V_{\bar{c}}^{-}$where $\operatorname{dim} V_{c}^{+}=n r-1$ and $\operatorname{dim} V_{\bar{c}}=1$. And setting $W^{\sigma, \pm}=U_{c} \otimes V_{\bar{c}}^{ \pm}$, we have the homomorphism of $K$ module

$$
\begin{aligned}
& W^{\sigma_{+}} \longrightarrow \mathfrak{p}^{-} \otimes W^{\sigma_{-}-} \\
& \cong U_{c} \otimes\left[V_{\boldsymbol{c}}^{+} \longrightarrow \mathfrak{p}^{-} \otimes V_{\boldsymbol{c}}^{-}\right] .
\end{aligned}
$$

which induces the $\sigma$-part of the first Gauss-Manin complex on $M$

$$
\nabla_{\sigma}: \mathscr{I}_{\sigma}^{0} \longrightarrow \Omega_{M}^{1} \otimes G r_{\Phi_{\sigma}}^{-1},
$$

where we set $\mathscr{F}_{\sigma}^{p}=\mathcal{O}_{M}\left(\boldsymbol{W}_{\boldsymbol{C}}^{\sigma}\right) \cap \mathscr{F}^{p}$. From Lemma (5.20), the homomorphism (6.26) is an isomorphism of $K$-modules and so the sheaf homomorphism (6.27) is also an isomorphism. Now let us write $\mathscr{D}=G_{R} / K$. Since $K$ is compact, $W^{\sigma,+}, \mathfrak{p}^{-}$ and $W^{\sigma,-}$ admit $G_{R^{-}}$invariant hermitian metrics, which induce hermitian metrics on the locally free sheaves $\mathscr{I}_{\sigma}^{0}, \Omega_{M}^{1}$ and $G r_{\Phi_{\sigma}}^{-1}$ respectively. Note that on $\mathscr{F}_{\sigma}^{0}$ 
and $G r_{\Phi_{\sigma}}^{-1}$ these metric are constant multiple of the metric induced by the original polarization $A$. Let $E$ be any locally free sheaf on $M=\Gamma \backslash \mathscr{D}$ induced by a $K$-representation with an above hermitian metric $h$. In [Mum], Mumford showed that such a $E$ admits a canonical extension $\bar{E}$ to a smooth toroidal compactification $\bar{M}$ in (6.1) such that $h$ is a singular hermitian metric good on $\bar{M}$. (For the definition of goodness of a singular hermitian metric, see [Mum, $\S 1]$.) One can see that such canonical extensions of $\mathscr{I}_{\sigma}^{0}$ and $G r_{q_{\sigma}}^{-1}$ coincides with $\overline{\mathscr{F}}_{\sigma}^{0}$ and $G r_{\overline{\mathscr{q}}_{\sigma}}^{-1}$ defined in (6.4), that is, those induced from the Deligne's canonical extension. (For the proof of this fact, see [H, Theorem 4.2].) Moreover, the canonical extension of $\Omega_{M}^{1}$ in the sense of Mumford is $\Omega_{M}^{1}(\log D)$. Therefore, by uniqueness of canonical extensions, the isomorphism (6.27) is extended to the isomorphism

$$
\bar{\nabla}_{\sigma}: \overline{\mathscr{\Psi}}_{\sigma}^{0} \longrightarrow \Omega_{\frac{1}{M}}^{1}(\log D) \otimes G r_{\bar{\Psi}_{\sigma}}^{-1},
$$

over $\bar{M}$. Then by (ii), Proposition (6.22), we have $H^{1}\left(\bar{M}, j_{*} \mathbb{W}_{c}^{\sigma}\right)^{0,0}=0$. From this, by the same argument as in Lemma (5.13), we deduce the vanishing condition (6.24), which implies the finiteness of the Mordell-Weil group.

Next we will deal with the case (6.2). In this case, $F_{1}=\boldsymbol{Q}$ and $G_{\boldsymbol{R}} \cong S U_{3}(\boldsymbol{H})^{-}$ $\cong S U(3,1, C)$. We use the same notation as in (5.23). By the same reason as in the case (6.3), we only have to show that $\boldsymbol{H}^{1}(\bar{M}, \bar{\nabla})=0$ where $\bar{\nabla}$ is the canonical extension of the Gauss-Manin complex. Over $M$, we have the isomorphism (5.27), so again by the uniqueness of the canonical extension, we have the isomorphism

$$
\text { coker } \bar{\nabla} \cong U_{C} \otimes S^{2}(\bar{I})
$$

where $\bar{I}$ is the canonical extension of the sheaf $\mathscr{I}$ (see (5.23)) to $\bar{M}$. As in proof of Proposition (5.28), we only have to show that $H^{0}\left(\bar{M}, S^{2}(\overline{\mathscr{C}})\right)=0$. As in (5.29), we have the inclusion

$$
H^{0}\left(\bar{M}, S^{2}(\bar{I})\right) \subset H^{0}\left(\bar{M}, S^{2} \overline{\left(T_{C}\right)}\right)
$$

where $\overline{\boldsymbol{T}}_{C}$ is the canonical extension of $\boldsymbol{T}_{C}$. We have the isomorphism $H^{0}(\bar{M}$, $\left.S^{2}\left(\overline{\boldsymbol{T}}_{C}\right)\right) \cong H^{0}\left(M, S^{2}\left(\boldsymbol{T}_{C}\right)\right) \quad($ see $[\operatorname{ShzY},(3.1 .1)])$, and by $(4.13) H^{0}\left(M, S^{2}\left(\boldsymbol{T}_{C}\right)\right)=0$. So we have the desired assertion.

(6.30) Remark. If $\operatorname{dim} \mathscr{D}=1$ and $M=\Gamma \backslash \mathscr{D}$ is not compact, the finiteness follows from the result in [Z1]. Let $f: \mathscr{X} \rightarrow M$ be a Kuga fiber space and $\bar{f}$ : $\bar{X} \rightarrow \bar{M}$ the semiabelian scheme in (6.4). By (6.8), we only have to prove that $H^{0}\left(\bar{M}, \mathcal{O}_{\bar{M}}^{a n}(\mathscr{X})\right)$ is finite. Then by (6.22) (cf. [Z1, Corollary (10.2)]), we have $H^{0}\left(\bar{M}, \mathcal{O}_{\bar{M}}^{\frac{a}{M}}(\overline{\mathscr{X}})\right) \cong H^{1}\left(\bar{M}, j_{*} \boldsymbol{W}_{Z}\right)^{0,0}$. Then [Z1, Lemma $\left.(12.4)\right]$ says that $H^{1}(\bar{M}$, $\left.i_{*} W_{C}\right)^{0,0}=0$, and hence the Mordell-Weil group is finite. 


\section{References}

[A] Artin, M., Néron Models, Arithmetic Geometry, Cornell, G., Silverman, J.H. eds., Springer-Verlag, 1986, pp. 213-230.

[Ba-B] Baily, Jr. W.L. and Borel, A., Compactification of arithmetic quotients of bounded symmetric domains, Ann. of Math., 84 (1966), 442-528.

[B] Borel, A., Regularization theorems in Lie algebra cohomology. Applications, Duke Math. J., 50 (1983), 602-624.

[B-C] Borel, A. and Casselman, W., $L_{2}$-cohomology of locally symmetric manifolds of finite volume, Duke Math. J., 50 (1983), 625-647.

[B-W] Borel, A. and Wallach, N., Continuous cohomolgy, discrete subgroups and representations of reductive groups, Ann. of Math. Studies, 94, Princeton Univ. Press, 1980.

[C-K-S] Cattani, E., Kaplan, A. and Schmid, W., $L_{2}$ and intersection cohomologies for a polarized variation of Hodge structure, Invent. Math., 87, 217-252.

[D1] Deligne, P., Equation différentielles à points singuliers réguliers, Lecture Notes in Math., 163 (1970), Springer-Verlag, Berlin-Heidelberg-New York.

[D2] - Théorie de Hodge, II, Publ. Math. IHES, 40 (1971), 5-57.

[G-M] Goresky, M. and MacPherson, R., Intersection homology II, Invent. Math., 72 (1983), 77-129.

[H] Harris, M., Functorial properties of toroidal compactifications of locally symmetric varieties, Proc. London Math. Soc., 59 (1989), 1-22.

[He] Hemperly, C.J., The parabolic contribution to the number of linear independent automorphic forms on certain bounded domain, Amer. J. Math., 94 (1972), 1078-1100.

[K-K] Kashiwara, K. and Kawai, T., The Poincaré lemma for variations of polarized Hodge structures, Publ. RIMS, Kyoto Univ., 23 (1987), 345-407.

[L] Looijenga, E., $L_{2}$-cohomology of locally symmetric varieties, Compositio Math., 67 (1988), 3-20.

[M-M] Matsushima, Y. and Murakami, S., On vector bundle valued harmonic forms and automorphic forms on symmetric Riemannian manifolds, Ann. of Math., 78 (1963), 365-416.

[Mo] Mok, Ngaiming, Aspect of Kähler geometry on arithmetic varieties, Preprint.

[Mo-T] Mok, Ngaiming and To, Wing-Keung, Eigensections of Kuga families of Abelian varieties and Finiteness of their Mordell-Weil groups, Preprint.

[Mum] Mumford, D., Hirzebruch's Proportionality Theorem in the Non-Compact Case, Invent. Math., 42 (1977), 239-272.

[S1] Satake, I., Algebraic structure of symmetric domains, Publ. Math. Soc. Japan, 14, Iwanami Shoten and Princeton University Press, 1980.

[S2] - Symplectic representations of algebraic groups satisfying a certain analyticity condition, Acta Math., 117 (1967), 215-279.

[Sa-St] Saper, L. and Stern, M., $L_{2}$-cohomology of arithmetic varieties, Ann. of Math., 132 (1990), 1-69.

[Sc] Schmid, W., Variation of Hodge structure: The singularities of the period mapping, Invent. Math., 22 (1973), 211-319.

[Sh1] Shimura, G., On analytic families of polarized abelian varieties and automorphic functions, Ann. of Math., 78 (1963), 149-192.

[Sh2] — - Moduli and fiber systems of abelian varieties, Ann. of Math., 83 (1966), 294-338.

[Sh3] - Discontinuous groups and abelian varieties, Math. Ann., 168 (1967), 171-199. 
[Sd] Shioda, T., On elliptic modular surfaces, J. Math. Soc. Japan, 24 (1972), 20-59.

[Shz] Shimizu, H., On discontinuous groups operating on product of the upper half planes, Ann. of Math., 77 (1963), 33-71.

[ShzY] Shimizu, Y., Mixed Hodge Structures on Cohomologies with Coefficients in a Polarized Variation of Hodge Structure, Adv. Stud. in Pure Math., 10 (1985), 695-716.

[Si1] Silverberg, A., Mordell-Weil groups of generic Abelian varieties, Invent. Math., 81 (1985), 71-106.

[Si2] Cohomology of fiber systems and Mordell-Weil groups of Abelian Varieties, Duke Math. J., 56 (1988), 41-46.

[Si3] , Moredell-Weil groups of generic abelian varieties in the unitary case, Proc. of the A.M.S., 104 (1988), 723-728.

[W] Weil, A., Adeles and algebraic groups, Notes by M. Demazure and T. Ono, IAS, Princeton, N. J., 1961.

[Z1] Zucker, S., Hodge theory with degenerating coefficients: $L_{2}$-cohomology in the Poincaré metric, Ann. of Math., 109 (1979), 415-476.

[Z2] , Locally homogeneous variations of Hodge structure, L'Ens. Math., 27 (1981), 243-276.

[Z3] - Generalized Intermediate Jacobians and the Theorem on Normal Functions, Invent. Math., 33 (1976), 185-222. 